\title{
Probabilistic approach to cloud and snow detection on Advanced Very High Resolution Radiometer (AVHRR) imagery
}

\author{
J. P. Musial ${ }^{1,2, *}$, F. Hüsler ${ }^{1,2}$, M. Sütterlin ${ }^{1,2}$, C. Neuhaus ${ }^{1,2}$, and S. Wunderle ${ }^{1,2}$ \\ ${ }^{1}$ Geographisches Institut der Universität Bern (GIUB), 3012 Bern, Switzerland \\ ${ }^{2}$ Oescher-Zentrum für Klimaforschung, Universität Bern, Zähringerstrasse 25, 3012, Bern, Switzerland \\ * currently at: the Remote Sensing Department, Institute of Geodesy and Cartography, Modzelewskiego 27, \\ 02-679 Warsaw, Poland
}

Correspondence to: J. P. Musial (jan.musial@giub.unibe.ch)

Received: 15 May 2013 - Published in Atmos. Meas. Tech. Discuss.: 25 September 2013

Revised: 28 January 2014 - Accepted: 3 February 2014 - Published: 28 March 2014

\begin{abstract}
Derivation of probability estimates complementary to geophysical data sets has gained special attention over the last years. Information about a confidence level of provided physical quantities is required to construct an error budget of higher-level products and to correctly interpret final results of a particular analysis. Regarding the generation of products based on satellite data a common input consists of a cloud mask which allows discrimination between surface and cloud signals. Further the surface information is divided between snow and snow-free components. At any step of this discrimination process a misclassification in a cloud/snow mask propagates to higher-level products and may alter their usability. Within this scope a novel probabilistic cloud mask (PCM) algorithm suited for the $1 \mathrm{~km} \times 1 \mathrm{~km}$ Advanced Very High Resolution Radiometer (AVHRR) data is proposed which provides three types of probability estimates between: cloudy/clear-sky, cloudy/snow and clearsky/snow conditions. As opposed to the majority of available techniques which are usually based on the decision-tree approach in the PCM algorithm all spectral, angular and ancillary information is used in a single step to retrieve probability estimates from the precomputed look-up tables (LUTs). Moreover, the issue of derivation of a single threshold value for a spectral test was overcome by the concept of multidimensional information space which is divided into small bins by an extensive set of intervals. The discrimination between snow and ice clouds and detection of broken, thin clouds was enhanced by means of the invariant coordinate system (ICS) transformation. The study area covers a wide range
\end{abstract}

of environmental conditions spanning from Iceland through central Europe to northern parts of Africa which exhibit diverse difficulties for cloud/snow masking algorithms. The retrieved PCM cloud classification was compared to the Polar Platform System (PPS) version 2012 and Moderate Resolution Imaging Spectroradiometer (MODIS) collection 6 cloud masks, SYNOP (surface synoptic observations) weather reports, Cloud-Aerosol Lidar and Infrared Pathfinder Satellite Observations (CALIPSO) vertical feature mask version 3 and to MODIS collection 5 snow mask. The outcomes of conducted analyses proved fine detection skills of the PCM method with results comparable to or better than the reference PPS algorithm.

\section{Introduction}

Cloud and snow detection on satellite imagery is a common part of a wide range of geophysical analysis. Therefore any misclassification introduced at this step have a direct effect on study results and may alter the final conclusions (GómezChova et al., 2007). This issue has been widely discussed by a number of authors: Jones et al. (1996) described a significant diurnal bias in the $0.5^{\circ}$ spatially averaged AlongTrack Scanning Radiometer (ATSR) sea surface temperature product over the South Atlantic induced by the residual cloud contamination; Kaufman et al. (2005) found that misclassified clouds in Moderate Resolution Imaging Spectroradiometer (MODIS) imagery lead to 0.02 bias in the aerosol 
optical thickness (AOT) estimates; Hall and Riggs (2007) analysed the improvements of cloud/snow discrimination in MODIS collection 5 data sets; Pincus et al. (2012) discussed the differences between cloud climatologies derived from MODIS and International Satellite Cloud Climatology Project (ISCPP) data sets induced by different detection sensitivities and treatment of thin cirrus and partially cloudy pixels. Furthermore inconsistencies in satellite products employed by climate models increase their variability, which mostly originates from the parametrisation of cloud radiative forcing (Houghton et al., 1996). In this respect accurate discrimination of cloud- and snow-covered areas supported by uncertainty estimations is required. Some of the existing approaches (Ackerman et al., 1998; Derrien and Le Gléau, 2005; Khlopenkov and Trishchenko, 2007; Vemury et al., 2001) separate classification results into few confidence categories (e.g. clear, probably clear, probably cloudy, cloudy). Nevertheless, implementation of this qualitative information into an error budget calculation of higher-level products is not straightforward. The solution to this problem involves derivation of continuous probability estimates of each pixel belonging to clear, cloudy and preferably snow classes. There are few existing approaches which provide such a quantitative probability distribution together with classification results. Some of them are based on classical (Merchant et al., 2005; Uddstrom et al., 1999) or naïve (Heidinger et al., 2012) Bayesian theories which combine results of a single classifier (i.e. spectral/textural test) with a priori assumption on cloud condition in order to obtain posterior classification probability. The a priori knowledge originates from additional data sets (e.g. climate model outputs) or collocated satellite observations (e.g. Cloud-Aerosol Lidar and Infrared Pathfinder Satellite Observations (CALIPSO)). Another algorithm proposed by Plummer (2008) expresses probability of cloud discrimination as a distance between tests results and threshold values. The final estimate is deemed as a maximum probability value across all performed tests. Another cloud masking method (Tian et al., 2000) involves probabilistic neural network classifiers employed to analyse temporal changes in a sequence of images. The clustering methods based on the expectation-maximisation (EM) technique were also found to be suitable for cloud probability retrieval (Gómez-Chova et al., 2007).

Derivation of probability estimates for snow discrimination on satellite imagery has been even less explored than in the case of cloud detection. Recently, Hüsler et al. (2012) modified the aggregated rating approach proposed by Khlopenkov and Trishchenko (2007) to suite European Alpine area and computed posterior snow classification probabilities employing logistic regression between ground data and numerical scores generated by spectral tests.

The main aim of this study was to develop a robust - that is, accurate and computationally inexpensive - algorithm that provides consistent probability estimates of a particular pixel in a satellite scene belonging to clear-sky, cloudy or snow classes. As the name "probabilistic cloud mask" (PCM) suggests, the main focus of this study is on cloud coverage; however the validation of the snow component is presented as well. The PCM algorithm is suited for the $1 \mathrm{~km} \times 1 \mathrm{~km}$ Advanced Very High Resolution Radiometer (AVHRR) local area coverage (LAC) data covering the extensive European region spanning from the northern parts of Africa to Iceland and the northernmost regions of Norway. The selected study area encompasses a wide range of ecosystems from desert to boreal vegetation and perennial snow together with broad illumination conditions including polar day and night. The variety of environmental conditions reflects different challenges occurring during the satellite cloud and snow discrimination.

The next section gives a short overview on existing algorithms for cloud and snow detection on AVHRR imagery with an emphasis on required data sets and types of tests applied. Section 3 describes principles of the PCM method beginning with the reasoning for the spectral features enhancement by means of the invariant coordinate system (ICS) transformation. Further the concept of multidimensional information space is discussed and the set of required input data together with binning values are introduced. Next, the proposed methodology and its numerical implementation are discussed together with the theoretical algorithm limitations. Section 4 presents the comparison between the PCM results and the reference data consisting of PPS (Polar Platform System) and MOD35 (MODIS) cloud masks, MOD10A1 (MODIS) daily snow mask, CALIPSO vertical feature mask and SYNOP (surface synoptic observations) cloud observations. Section 5 contains an analysis of the acquired results, which are concluded in Sect. 6.

\section{Overview of existing multi-thresholding algorithms for satellite cloud and snow detection}

The majority of existing algorithms for cloud and snow detection incorporate a series of spectral, textural and/or temporal tests (often called features) which are arranged in a decision-tree scheme. Singular tests are based on a comparison of radiances from a spectral channel or combination of channels with a threshold value which could be static (Bernstein, 1982) or may vary together with angular and atmospheric conditions (Ackerman et al., 1998; Dybbroe et al., 2005a; Di Vittorio and Emery, 2002; Yang et al., 2007). The latter approach is more robust as the strong influence of atmospheric concentration of gases and aerosols, bidirectional reflectance, and acquisition geometry on satellite measurements has been widely discussed in the literature (Fraser and Kaufman, 1985; Saunders et al., 1999; Schaaf et al., 2002; Vermote et al., 1997). Further surface properties such as albedo and emissivity modify spectral contrast and influence the cloud/snow detection sensitivity (Minnis et al., 2008; Yhann and Simpson, 1995). It is apparent over dark 
surfaces, such as water in the near-infrared (NIR) spectrum, where discrimination of clouds is easier than over bright surfaces like deserts or snow (Ackerman et al., 1998; Di Vittorio and Emery, 2002). This implies that the thresholds should also vary across different land cover types. More difficult situations for the accurate classification are related to pixels partially covered by snow or thin/broken clouds, where there is a strong contribution from the surface in the measured satellite signal (Simpson et al., 2001). Furthermore, high aerosol loads in the atmosphere are often misclassified as cloud due to similar spectral signature (Martins et al., 2002). In order to account for all of the mentioned factors, the threshold parametrisation is either derived empirically or it is optimised by means of the radiative transfer (RT) modelling. Afterwards it is stored as look-up tables (LUTs) to save computation time during the cloud/snow masking process (Dybbroe et al., 2005a).

Usually a single test provides a binary state of a pixel, such as cloudy/clear-sky or snow/snow-free. Further this information might be treated in a variety of ways. Some algorithms (Key and Barry, 1989) report cloud whenever one of the tests performed has been successful. Other methods (Dybbroe et al., 2005a; Derrien and Le Gléau, 2005) arrange features in groups with decreasing detection sensitivity. In order to mark a pixel as cloudy, all tests within a group have to be successful. Alternative approaches are based on a fuzzy logic, where the final confidence estimate of a pixel state is expressed as a product of singular estimates for each group of tests (Ackerman et al., 1998) or as a total sum of score values (Khlopenkov and Trishchenko, 2007; Hüsler et al., 2012). Nevertheless, during the further classification process these continuous confidence estimates are transformed into discrete classes using threshold values.

\subsection{Ancillary data employed by multi-thresholding algorithms}

Cloud and snow discrimination on satellite imagery is mainly based on multi-spectral measurements; however, additional ancillary data are required for a threshold parametrisation. This complementary information might be divided into meteorological and surface data sets, the first of which feature high temporal variations, whereas the latter ones usually are stable over time or change accordingly to well-known daily and annual cycles (Yhann and Simpson, 1995).

\subsubsection{Ancillary meteorological data}

An instantaneous atmospheric state can be estimated either by climate models or by rough approximations based on climatological mean values. Usually, such simulations are of low spatial resolution, yet an interpolation to a satellite grid results in significant bias over areas with rough topography (Zhao et al., 2008) or around zones with high temperature gradients, such as coastlines. Another source of inaccuracies is temporal sampling of a climate model which may not correspond to satellite acquisition time; thus data interpolation between two model steps is required (Khlopenkov and Trishchenko, 2007; Minnis et al., 2008).

The RT transfer within the atmosphere is significantly altered by the concentration of water vapour often denoted as total column water vapour (TCWV). It expresses the integrated mass of water vapour per cross-sectional area unit of an atmospheric column $\left(\mathrm{kg} \mathrm{m}^{-2}\right)$. Instead of TCWV, some of the sophisticated satellite cloud detection algorithms (Minnis et al., 2008) utilise humidity, temperature and wind profiles to more accurately resolve the RT processes. Furthermore, the absorption of radiation by water vapour within the atmosphere is strongly related to the optical path, which in turns depends on the sensor viewing angle. Thus, instrument acquisition geometry should be taken into account during the cloud discrimination on satellite imagery (Yhann and Simpson, 1995). In cases when the estimates of atmospheric water vapour concentration are not available, one can utilise the split-window approach proposed by Saunders and Kriebel (1988) to improve detection of cirrus clouds. This technique is based on the LUT, where threshold values are stored as a function of $10.8-12.0 \mu \mathrm{m}$ brightness temperature difference (BTD), secant of the sensor viewing angle and $10.8 \mu \mathrm{m}$ brightness temperature (BT).

Thermal contrast between surface and cloud tops can be a decisive factor when the spectral information is not sufficient for confident discrimination between cloudy and clear-sky conditions. It is derived as a difference between skin temperature (SKT) obtained from a climate model and the $10.8 \mu \mathrm{m}$ BT. Moreover, the SKT data together with other atmospheric variables serve as inputs to RT calculations, which are used to simulate satellite signals measured at specific spectral ranges for clear-sky conditions. These expected values are then compared to real data acquired by sensor, and if the deviations are significant a cloud presence might be assumed. Another useful piece of information for the cloud detection on satellite imagery is a difference between air temperatures provided at two lowest altitude levels of a climate model. This indicates a presence of low-level temperature inversion which reverses the expected thermal signature of clouds and may lead to misclassification (Dybbroe et al., 2005a).

\subsubsection{Ancillary surface data}

Surface characteristics are of great importance for the cloud/snow discrimination on satellite imagery, especially the land cover categorisation. Therefore, availability of binary land/water mask is a minimum requirement for application of suitable threshold configuration within a classification algorithm. Sophisticated threshold parametrisations (Dybbroe et al., 2005a; Minnis et al., 2008) utilise more detailed land cover information together with digital elevation model (DEM) data to enhance detection accuracy over areas particularly ambiguous for correct classification (e.g. cloud 
detection over mountains, snow detection under the tree canopy). Additionally to land cover periodically updated snow/sea ice coverage data could be utilised for the further threshold refinements (Dybbroe et al., 2005a; Minnis et al., 2008; Ackerman et al., 1998). In order to model measured satellite signal, surface spectral properties such as albedo and emissivity have to be considered (Minnis et al., 2008). They change systematically over the coarse of a year; therefore the RT simulation together with threshold parametrisation should feature temporal sampling (Dybbroe et al., 2005a).

\subsection{Features used for satellite cloud/snow detection}

Discrimination of cloud and/or snow coverage on satellite imagery is based on specific spectral properties at particular wavelengths which are utilised regardless of the sensor and algorithm employed. Next Subsections describe in details those of them which are applicable to the AVHRR instrument.

\subsubsection{Reflectance tests in the 0.6 and $0.8 \mu \mathrm{m}$ bands}

In the visible (VIS) $0.6 \mu \mathrm{m}$ and NIR $0.8 \mu \mathrm{m}$ spectral regions clouds and snow appear much brighter than the underlying background. Furthermore, the spectral contrast of those surfaces over land is higher in the $0.6 \mu \mathrm{m}$ channel, whereas over water bodies it is more distinct in the $0.8 \mu \mathrm{m}$ channel. Nonetheless, discrimination between snow and cloud cover in both wavelengths is impossible; hence additional information either from the 1.6 or $3.7 \mu \mathrm{m}$ channel is required. In order to diminish the influence of illumination conditions on retrieved reflectance, it is divided by a cosine of Sun zenith angle (SZA) and adjusted for the Sun-Earth distance variations. These corrections should be applied to any channel data within the reflective part of the electromagnetic spectrum. Some approaches (Dybbroe et al., 2005a) utilise reflectance in the channel $0.6 \mu \mathrm{m}$ with and without the SZA normalisation, claiming that the latter is useful for cloud detection at extremely high SZA $\left(>86^{\circ}\right)$ when $\frac{\text { reflectance }}{\cos (\mathrm{SZA})} \rightarrow \infty$.

\subsubsection{Reflectance tests in the 1.6 and $3.7 \mu \mathrm{m}$ bands}

In the 1.6 and $3.7 \mu \mathrm{m}$ spectra there is a significant reflectance contrast between water clouds and snow. This feature together with the reflectance at $0.6 \mu \mathrm{m}$ is employed by the Normalized Difference Snow Index (NDSI) in order to standardise and enhance snow detection. Although high NDSI values are usually associated with snow-covered areas, they may refer to ice clouds as well. This ambiguity is difficult to resolve using only spectral information; thus some ancillary data such as SKT should be utilised. The presence of an ice cloud can be assumed when the difference between SKT and the $10.8 \mu \mathrm{m}$ BT significantly deviates from $0 \mathrm{~K}$. This situation often occurs due to strong convection during the summertime when cloud tops (e.g. cumulonimbus) consist of ice particles while the Earth's surface remains warm. Even during the cold part of a year this thermal contrast might be decisive depending on accuracy and temporal sampling of the SKT data.

\subsubsection{Brightness temperature difference test between 3.7 and $10.8 / 12.0 \mu \mathrm{m}$ bands}

Contamination of the $3.7 \mu \mathrm{m}$ signal by the solar component results in quite different day and night appearance of clouds at this wavelength. This is particularly apparent for water clouds which reflect a lot of the $3.7 \mu \mathrm{m}$ radiation during day leading to high BT, while during night they appear much colder. Due to this issue the $3.7-10.8$ or $3.7-12.0 \mu \mathrm{m}$ BTDs are used only during the night, when they are negative for optically thick clouds and positive for thin clouds. Furthermore, during night the 3.7-10.8 $\mu \mathrm{m}$ BTD was found to be more useful for detection of warm clouds and low stratus/fog layers (Eyre et al., 1984), whereas the difference between 3.7 and $12.0 \mu \mathrm{m}$ has high sensitivity to thin cirrus (Dybbroe et al., 2005a). For low radiative temperatures measured by early generations of the AVHRR instrument (prior to NOAA15) some cloud detection inconsistencies may occur due to periodic noise in the $3.7 \mu \mathrm{m}$ channel (Warren, 1989).

\subsubsection{Brightness temperature difference test between 10.8 and $12.0 \mu \mathrm{m}$ bands}

The 10.8-12.0 $\mu \mathrm{m}$ BTD is particularly useful for detection of cirrus clouds which are not apparent at other wavelengths. It is positive for thin clouds due to higher atmospheric transmittance at $10.8 \mu \mathrm{m}$ than at $12.0 \mu \mathrm{m}$ (Inoue, 1985). Moreover, it heavily depends on atmospheric water vapour concentration and sensor viewing angle. Therefore, a threshold value for the cirrus detection test should be derived dynamically using radiative transfer modelling or a robust parametrisation (Saunders and Kriebel, 1988).

\subsubsection{Temperature difference test between Earth's surface and the $10.8 \mu \mathrm{m}$ band}

The spectral region around $10.8 \mu \mathrm{m}$ is slightly affected by absorption of atmospheric gases (so-called atmospheric window); thus it approximates well the surface temperature at least in regions well outside of the tropics. If the thermal contrast between $10.8 \mu \mathrm{m} \mathrm{BT}$ and SKT data derived from climatological records or from climate models is sufficiently high, then the cloud presence can be assumed. This feature is particularly useful over ice-free ocean during night when water temperature is usually well approximated by the climate models. However, special attention is required in the case of temperature inversion, when the expected positive difference between SKT and $10.8 \mu \mathrm{m}$ cloud top temperature becomes negative. Over barren or sparsely vegetated areas such as deserts, a strong diurnal surface temperature cycle might be poorly represented in the SKT data (Pavolonis, 2010), which may lead to erroneous test results. Therefore, considering all 
of the mentioned aspects, threshold values for this test over land should be rather conservative and limited to detection of relatively cold clouds.

\subsubsection{Spatial uniformity tests}

Regardless of the spectral properties of clouds, their appearance on a satellite image is distinct especially over homogeneous surfaces (Saunders and Kriebel, 1988; Ackerman et al., 1998). If a local radiance variation analysed within a small image window (e.g. $5 \times 5$ pixels) is significant, then the cloud presence may be assumed over the central pixel. Due to high surface heterogeneity over land, this test is usually applied only over water bodies using $0.8 \mu \mathrm{m}$ channel during the day and $10.8 \mu \mathrm{m}$ channel for the night. When dealing with polar regions, the sea ice cover has to be considered as it may exhibit similar local radiance variations to clouds. In this respect, during the nighttime conditions the $3.7 \mu \mathrm{m}$ BT or 3.7-12 $\mu \mathrm{m}$ BTD are used to detect cloud edges and to filter out leads (cracks in ice filled with water) which could be of sub-pixel size (Dybbroe et al., 2005a).

\subsubsection{Temporal consistency tests}

Most of the high-frequency positive anomalies of surface albedo or negative thermal anomalies in a sequence of satellite images can be attributed to clouds. The temporal consistency tests require derivation and periodical update of reference clear-sky radiance statistics accumulated over some time for various spectral channels (Rossow and Garder, 1993). These calculations are usually performed over small spatial domains (e.g. $30 \mathrm{~km} \times 30 \mathrm{~km}$ ) for similar land cover types. More sophisticated approaches (Lyapustin et al., 2008) utilise the spatio-temporal covariance matrix in order to detect cloud-induced changes in spectral and textural signatures at a single pixel level. While comparing the clear-sky radiances with different acquisition times, special attention has to be paid to angular and atmospheric effects which alter the surface spectral response. To mitigate their impact, several techniques have been developed: threshold relaxation in order to account for a wide range of conditions; comparison of measurements taken at similar solar times from corresponding orbits; and atmospheric and bidirectional effects' corrections of the reference clear-sky composite. Additional data processing employed by the temporal consistency tests increases computational demands, yet they feature valuable detection skills especially for the geostationary satellites.

\subsection{Limitations of multi-spectral thresholding algorithms}

Detection of clouds and snow on satellite imagery by means of the multi-spectral thresholding algorithms usually requires estimation of a single threshold value for each test which accounts for a wide range of atmospheric, angular and surface conditions (Yang et al., 2007; Yhann and Simpson, 1995).
This complexity results either in reduced classification accuracy due to the threshold relaxation or in extensive analysis of spectral and ancillary information. However, in each situation any misplacement of a single threshold value leads to errors which could be depreciated by utilisation of a fuzzy approach (see score functions in Khlopenkov and Trishchenko, 2007). Furthermore, parametrisations of the multi-spectral thresholding algorithms are mostly derived empirically; thus their application to other satellite sensors is not straightforward. Test sequence and design of a decision-tree method have crucial significance because results of previous steps have an influence on the following ones. Thus any misclassification at the level of a single test/group of tests may mislead the algorithm and lead to incorrect results.

\section{Probabilistic cloud mask (PCM) algorithm description}

The main scientific motivation for the PCM algorithm development was to create a robust (meaning accurate and fast) classification method, which would diminish the main sources of errors originating from the commonly used multispectral thresholding approach (see Sect. 2.3). It was supposed to detect clouds and snow cover on AVHRR imagery and to provide classification probability estimates. The PCM method stems from the multidimensional analysis of spectral features and ancillary data. Its parametrisation is derived on the basis of training data sets composed of binary cloud and snow masks. In this way, results of two discrimination algorithms are combined and supplemented with probability estimates without the lost of classification accuracy (see Sect. 4). The described features of the PCM method are unique amongst other available techniques which substantiate the need for its development.

In the next subsections the description of the PCM algorithm will be presented in the following order: input data utilised in the study, principles of the spectral features, concept of the multidimensional information space, methodology of classification, numerical implementation and postclassification with cloud shadow estimation.

\subsection{Required input data}

The PCM algorithm is suited for the AVHRR instrument, which has been operating aboard the suite of National Oceanic and Atmospheric Administration (NOAA) and Meteorological Operating (MetOp) polar-orbiting satellites. The selection of this sensor was related to its long data record (30 yr), which serves as a valuable input to short-range climatological studies conducted by the Remote Sensing Group of the University of Bern (Hüsler et al., 2011). The proposed (NOAA15 and later, MetOp series) instruments with spectral bands centred around $0.6,0.8$ and 1.6 or $3.7,10.8$ and $12.0 \mu \mathrm{m}$. For the AVHRR-3 sensors channel switching occurs 
at the illumination transition zone; for example the $3.7 \mu \mathrm{m}$ measurements are taken during the night while for the sunlit portion of an orbit the channel 1.6 is optionally activated (for some satellites, e.g. NOAA18 and NOAA19, over Europe this channel is always deactivated). However, the AVHRR-2 instruments are equipped only with the $3.7 \mu \mathrm{m}$ channel. Radiances at $1.6 \mu \mathrm{m}$ include only the solar component of the electromagnetic spectrum, whereas at $3.7 \mu \mathrm{m}$ most of the radiation originates from the Earth's surface with a small contribution of the solar signal. Nevertheless, this reflective part was approximated from the $3.7 \mu \mathrm{m}$ channel by subtracting thermal component approximated by the $10.8 \mu \mathrm{m}$ BT under the assumption of unit emissivity (Allen et al., 1990; Khlopenkov and Trishchenko, 2007).

In this study the $1 \mathrm{~km} \times 1 \mathrm{~km}$ LAC AVHRR measurements covering the extensive European subset $\left(-34^{\circ} \mathrm{W}\right.$ to $46^{\circ} \mathrm{E}$, $28^{\circ} \mathrm{N}$ to $71^{\circ} \mathrm{N}$ ) were utilised. During the algorithm training phase more than 2000 scenes with the $3.7 \mu \mathrm{m}$ channel configuration acquired by the NOAA16, 17, 18, and 19 satellites throughout the years 2009-2011 were employed, whereas for the $1.6 \mu \mathrm{m}$ channel configuration around 400 NOAA17 scenes from the year 2009 were used. As a result, a set of LUTs was derived which was further utilised by the PCM procedure to classify a collection of NOAA16 images from the year 2011 and a collection of NOAA17 and 18 images from the year 2008. These data sets were taken as an input to all presented analyses in this study. Due to the data availability issues time of the training data set for the NOAA16 satellite overlays with the analysis period. Nevertheless, the results for all of the selected NOAA platforms stay in a good agreement, which indicates that the overlapping period did not have a significant influence on the computed statistics.

Apart from the satellite measurements the following ancillary data were used: land cover obtained from the Global Land Cover 2000 Project (Mayaux et al., 2004; Bartholomé and Belward, 2005), DEM provided by the United States Geological Survey (USGS), and the SKT derived from the ECMWF (European Centre for Medium-Range Weather Forecasts) deterministic forecast with the $3 \mathrm{~h}$ step. All data sets were remapped to the Lambert equal-area projection which maintains the pixel size and thus is more suitable for spatial statistics calculations.

\subsection{Spectral features employed in the PCM method}

In the majority of cloud/snow masking techniques the multispectral information is selectively exploited through a sequence of independent tests. However, when few tests give opposite results the final decision can be ambiguous. In the PCM algorithm this issue is resolved by the concept of multidimensional LUT (see Sect. 3.3) which holds spectral and ancillary information together. Thus, for a particular data combination there is only one possible solution which eliminates the ambiguity between different test results. The size of the LUT is a limiting factor; therefore to reduce its dimensionality the ICS transformation is applied (Nordhausen et al., 2008; Tyler et al., 2009). It utilises the principal component analyses (PCA) (Mardia et al., 1979) and two scatter matrices in order to construct independent components which do not rely on a distribution mean. The first scatter matrix is a regular covariance matrix used to standardise data, while the second one is a matrix of the fourth moment (kurtosis) which describes data rotation within the PCA. The eigenvalue decomposition is performed on one matrix in a relation to the other one which results in the affine invariant co-ordinate system for multivariate observations. The matrices are derived on the basis of a randomly selected winter satellite scene with vast snow cover and utilised throughout the rest of transformations. To save computation time the ICS technique is performed only for the daytime data to combine reflectances with the thermal contrast between SKT and the $10.8 \mu \mathrm{m}$ BT. It is applied selectively to pixels with probable cloud contamination (high thermal contrast) which fulfil specific criteria. These restriction are meant to improve ice cloud detection over snow and broken cloud discrimination where spectral information is ambiguous, but thermal contrast with surface is significant. In this way spectral signatures of areas with small thermal contrast, which may be related to climate model inaccuracy (and not to presence of clouds), remain unchanged. In this respect ICS transformation over water bodies (areas further than $8 \mathrm{~km}$ from the shoreline) is performed for pixels with the SKT$10.8 \mu \mathrm{m}$ greater than $8 \mathrm{~K}$ to account for warm ocean currents not included in the SKT data. For the shoreline zones, it is applied to pixels with the $0.6 \mu \mathrm{m}$ reflectance higher than 0.3 to account for mixed land/water pixels. Furthermore, regions below $1200 \mathrm{~m}$ are only considered if the SKT $-10.8 \mu \mathrm{m}$ is greater than $8 \mathrm{~K}$. For higher altitudes this threshold is set to $16 \mathrm{~K}$ to account for the local thermal variations which cannot be resolved by a coarse-resolution climate model. Over land, pixels which are unlikely to be overcast with the reflectance lower than 0.15 at $0.6 \mu \mathrm{m}$ or with the $10.8 \mu \mathrm{m}$ BT greater than $290 \mathrm{~K}$ are not considered. After the ICS transformation the size of the LUT is reduced by the SKT dimension. Moreover, if it is not available, the PCM algorithm can still proceed without enhanced reflectances. A short overview on the spectral features employed in the PCM is presented in the next subsections.

\subsubsection{First enhanced spectral feature}

The first enhanced spectral feature consists of two components depending on the time of the day. For the sunlit portion of a scene limited by the SZA of $89^{\circ}$ it is composed of the second invariant component of the ICS transformation based on the SKT-10.8 $\mu \mathrm{m}$ temperature difference and on the reflectance at $0.6 \mu \mathrm{m}$ over land and at $0.8 \mu \mathrm{m}$ over water. This results in the enhanced spectral contrast because thin cirrus or cold sub-pixel clouds modify thermal signal more efficiently than the short-wave radiation (see Fig. 1). For the 


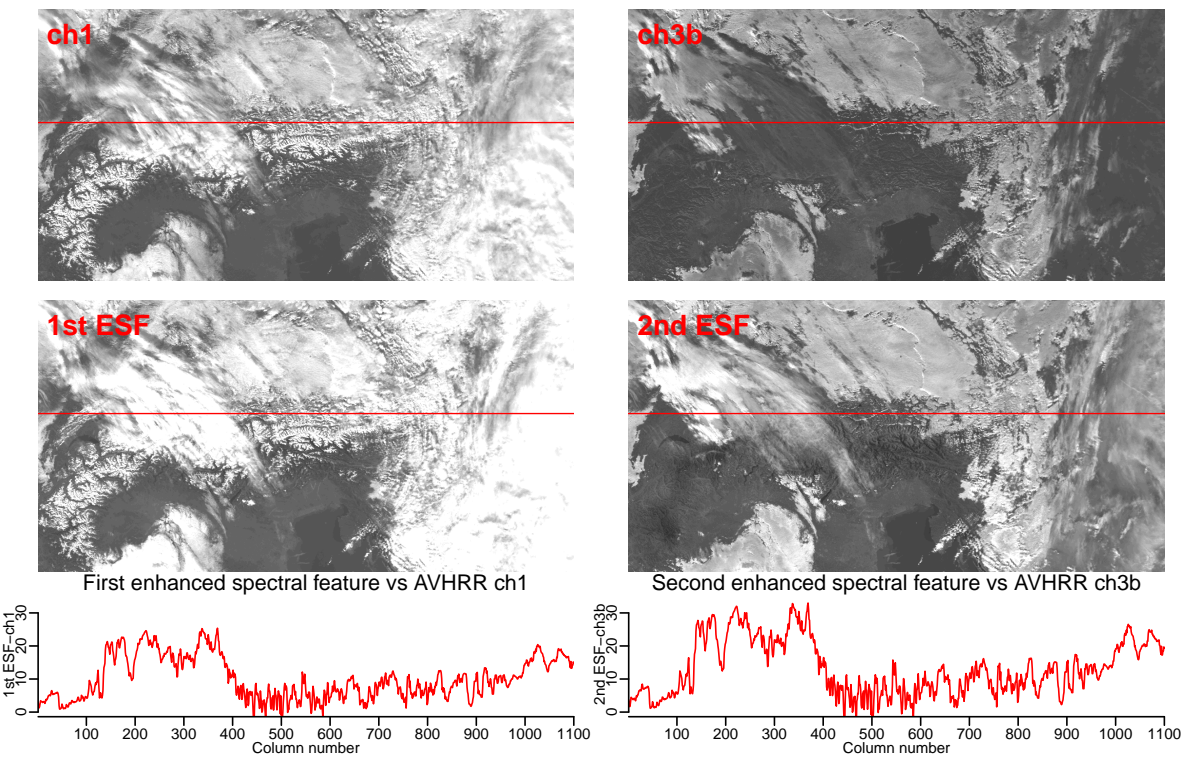

Fig. 1. Differences between enhanced spectral features (ESFs) generated by the invariant coordinate system (ICS) transformation and the AVHRR channel $1(0.6 \mu \mathrm{m})$ and channel $3 \mathrm{~b}(3.7 \mu \mathrm{m})$. Bottom panels depict the differences between the ESFs and the AVHRR reflectances retrieved from the spectral profiles marked as the red lines. For details see Sects. 3.2.2 and 3.2.1.

night part of a scene $\left(\mathrm{SZA} \geq 89^{\circ}\right)$ this feature consists of the scaled SKT-10.8 $\mu \mathrm{m}$ difference.

\subsubsection{Second enhanced spectral feature}

Content of the second enhanced spectral feature depends on the channel configuration of the AVHRR sensor and on the time of the day.

- Channel $1.6 \mu \mathrm{m}$ : whenever the channel $1.6 \mu \mathrm{m}$ is activated over the sunlit portion of a scene, this feature consists of the second invariant component of the ICS transformation based on the SKT- $10.8 \mu \mathrm{m}$ temperature difference combined with the reflectance at $1.6 \mu \mathrm{m}$. In this situation the thermal difference improves discrimination between ice cloud and snow which have very similar spectral signature at this wavelength. However, due to the fact that ice clouds have usually lower temperatures than the snow-covered land the SKT$10.8 \mu \mathrm{m}$ thermal contrast is often higher for clouds than for cloud-free snow-covered areas. For the nighttime part of a scene $\left(\mathrm{SZA} \geq 89^{\circ}\right)$ this feature does not contain any information.

- Channel $3.7 \mu \mathrm{m}$ : whenever the $3.7 \mu \mathrm{m}$ channel is activated, the second enhanced spectral feature is divided into two parts. The first one is computed for the SZA below $85^{\circ}$ and consists of the second invariant component of the ICS transformation based on the reflective part of the $3.7 \mu \mathrm{m}$ spectrum and the SKT$10.8 \mu \mathrm{m}$ difference. A combination of the reflectance with the thermal difference information enhances the ice cloud/snow discrimination in the same manner as described before (see Fig. 1). The $3.7 \mu \mathrm{m}$ reflectance for the $\mathrm{SZA}>85^{\circ}$ has a weak signal and contains a lot of noise. Thus the scaled 10.8-3.7 $\mu \mathrm{m}$ BTD is used for the twilight conditions $\left(85^{\circ} \geq \mathrm{SZA}<89^{\circ}\right)$ and for the nighttime.

\subsubsection{Third spectral feature}

The third spectral feature is not processed by the ICS transformation and is composed of the 10.8-12.0 $\mu \mathrm{m} \mathrm{BTD,} \mathrm{which}$ is useful for the thin cirrus cloud detection.

\subsection{Multidimensional information space}

The main concept of the PCM algorithm is based on the LUT with precomputed classification probability estimates in the form of an array composed of multiple dimensions corresponding to different spectral features, land cover classes and angular conditions. This array is called multidimensional information space because it holds spectral and ancillary information together as opposed to the spectral space, which contains only satellite measurements. In order to include continuous data such as spectral features or viewing angles in the array, they are binned by an extensive set of intervals. In the domain of signal processing this procedure corresponds to data quantisation (many-to-few mapping). Such an approach allows specifying multiple irregularly distributed binning values per feature, which resolves the problem of a single threshold estimation existing in the multi-spectral thresholding methods. Another advantage of the PCM algorithm is the direct derivation of probabilities from the LUT by means 
of all available spectral and ancillary information. Therefore all data are analysed at once in contrast to the decision-tree algorithms where features/tests are organised in a sequence.

The information space is composed of 8 dimensions:

- Time of the day divided into day $\left(\mathrm{SZA}<85^{\circ}\right)$, twilight $\left(\mathrm{SZA} \geq 85^{\circ}\right.$ and $\left.\mathrm{SZA}<89^{\circ}\right)$ and night $\left(\mathrm{SZA} \geq 89^{\circ}\right)$.

- First enhanced spectral feature divided by the following binning values: $0,0.025,0.05,0.075,0.1,0.125$, $0.15,0.175,0.2,0.225,0.25,0.275,0.30,0.35,0.40$, $0.45,0.50,0.60,0.80$ and 1.0 .

- Second enhanced spectral feature:

- $1.6 \mu \mathrm{m}$ channel activated: this feature is divided by the following binning values: $0,0.05,0.075$, $0.1,0.125,0.15,0.175,0.2,0.225,0.25,0.30$, $0.40,0.50,0.6$ and 0.7 .

- $3.7 \mu \mathrm{m}$ channel activated: this feature is divided by the following binning values: $0,0.025,0.05$, $0.075,0.1,0.125,0.15,0.175,0.2,0.225,0.25$, $0.30,0.40$ and 0.50 .

- Third spectral feature divided by the following binning values: $-0.8,-0.6,-0.4,-0.2,0.0,0.1,0.4,0.6,0.8$, $1.0,1.25,1.5,1.6,1.8,2.0,2.5,3.0$ and 3.5 .

- Texture feature, which is derived only over open waters by convolution of the $0.8 \mu \mathrm{m}$ reflectance for the sunlit areas and the $10.8 \mu \mathrm{m}$ BT during the nighttime with a $3 \times 3$ kernel (Eq. 1). The convolution results are scaled to $0-1$ range, where high values characterise pixels located at the cloud edges. This feature is divided by the following binning values: $0.05,0.1,0.25,0.5$ and 1 .

$$
\mathbf{K}=\left[\begin{array}{rrr}
-1 & -1 & -1 \\
-1 & 8 & -1 \\
-1 & -1 & -1
\end{array}\right]
$$

- Sensor viewing sectors, which are derived by dividing the satellite zenith angle with the following binning values: $0,15,30,45,55$ and 70 .

- Relative azimuth sectors, which are derived by dividing the relative azimuth (RAZ) angle, defined as a difference between Sun and sensor azimuth angles $\left(180^{\circ}=\right.$ forward scattering $)$, with the following binning values: $0,45,90,135$ and 180 .

- Land cover/use developed within the scope of the Global Land Cover 2000 (GLC2000) project (Bartholomé and Belward, 2005). There are three additional surface categories which are derived internally by the PCM algorithm: coastline water defined as a $8 \mathrm{~km}$ buffer zone from the shore including inland waters, Sun glint over water and Sun glint over desert, which are discriminated by the simple Sun-sensor angular dependency described by Ackerman et al. (1998). All these areas feature significantly higher reflectances due to specific angular conditions (Sun glint); higher concentration of non-maritime aerosols (Wang and Shi, 2006), sediments or algae (Wang and Shi, 2005) and shoreline variation induced naturally by tides or artificially by satellite geolocation problems.

The positions of values within the information space depend on diurnal and annual cycles. The first one is driven by the illumination conditions and alters mainly the reflectance due to the bidirectional effects. Therefore separate information spaces were developed for different satellite overpass times. For morning satellites with the $1.6 \mu \mathrm{m}$ channel activated, acquisition time was divided into two ranges: 00:0012:00 and 13:00-00:00 UTC. For the satellites with the $3.7 \mu \mathrm{m}$ channel activated the division was set to 07:00-10:00, 11:00-14:00, 15:00-18:00, and 19:00-06:00 UTC. The annual cycle is related to changes in albedo and surface emissivity properties induced by vegetation development and soil moisture variations. Thus the information spaces were further divided between different seasons: winter (NovemberJanuary), spring (February-April), summer (May-July), and autumn (August-October). Each possible daytime-season combination was stored separately for the 1.6 and $3.7 \mu \mathrm{m}$ channel configurations, which results in 48 LUTs.

$$
P \in\left\{\begin{array}{r}
0 \leq P \leq 100 \text { probability of } 0-100 \% \text { between } \\
\text { clear-sky and snow conditions }
\end{array}\right.
$$

\subsection{Methodology of classification}

In order to discriminate snow, clear-sky and cloudy pixels on a satellite image with the PCM algorithm, the information space has to be filled with classification probability estimates. This process is divided into 2 steps. First, a temporary information space is created which contains one more dimension where the categories clear-sky, cloudy and snow originating from the training data set are stored. Second, the frequencies of occurrence of those classes within each bin are transformed into the probability estimates. Development of the PCM method consists of the following steps:

1. Composition of training data set, which involves collocation of the AVHRR measurements with the snow/clear-sky/cloudy classification which may originate from ground data, supplementary satellite data source (e.g. CALIPSO and MODIS products) or from results of another classification algorithm applied to the AVHRR data. Nevertheless, the data quantity should be large enough to sufficiently sample the multidimensional array with information acquired under a 
wide range of environmental conditions. In this study the training data set was composed of the PPS version 2012 cloud masks (Dybbroe et al., 2005a) retrieved from AVHRR data and merged with the MOD10A1 daily snow masks (Hall et al., 2002) based on MODIS data. Those algorithms were selected due to their high classification accuracy (Dybbroe et al., 2005b; Hall and Riggs, 2007), as well as the data (MOD10A1) and source code (PPS) availability. It has to be emphasised that the mean PCM classification accuracy cannot exceed the one associated with the training data set. To improve the quality of the training data set, it was first visually inspected and low-quality scenes were removed. Then significant misclassifications occurring in the remaining images were edited and corrected by an analyst. This procedure was applied to the extensive data set $(\sim 2400$ scenes $)$ acquired over Europe between 2009 and 2011 by different AVHRR sensors mounted aboard NOAA satellites denoted with numbers $16,17,18$ and 19.

2. Formation of temporary information spaces, which involves computation of all spectral and textural features for the selected AVHRR training images. Further, the frequencies of occurrence of clear-sky, snow and cloudy classes originating from the training data for each combination of dimensions (i.e. features, angular and ancillary data) are inserted into the information space. Therefore, a single value within the temporary information space corresponds to numerical count of all pixels embedded into a nine-dimensional bin composed of the following elements: time of the day, three spectral features, texture feature, viewing and azimuth sectors, land cover, and PPS/MOD10A classification. In other words this array might be treated as a ninedimensional histogram. This procedure was repeated 48 times for each information space characterised by the different channel configuration $(1.6 / 3.7 \mu \mathrm{m})$, acquisition hour and season.

3. Derivation of classification probability, which is based on numerical counts from the temporary information spaces, which are first rearranged in descending order for every multidimensional bin. Further, the probability is computed as a simple ratio between counts of the most frequent classification category within a bin and the total number of counts in this bin. The retrieved value is described as a classification probability between the two most frequent classes within the bin. Similar analysis in a two-dimensional space is presented in Fig. 2. The PCM classification probability estimates are related to a combination of the following categories: snow-free/snow, clear-sky/cloudy, snow/cloudy. For the sake of visualisation obtained values are recoded according to Eq. (2). For bins which

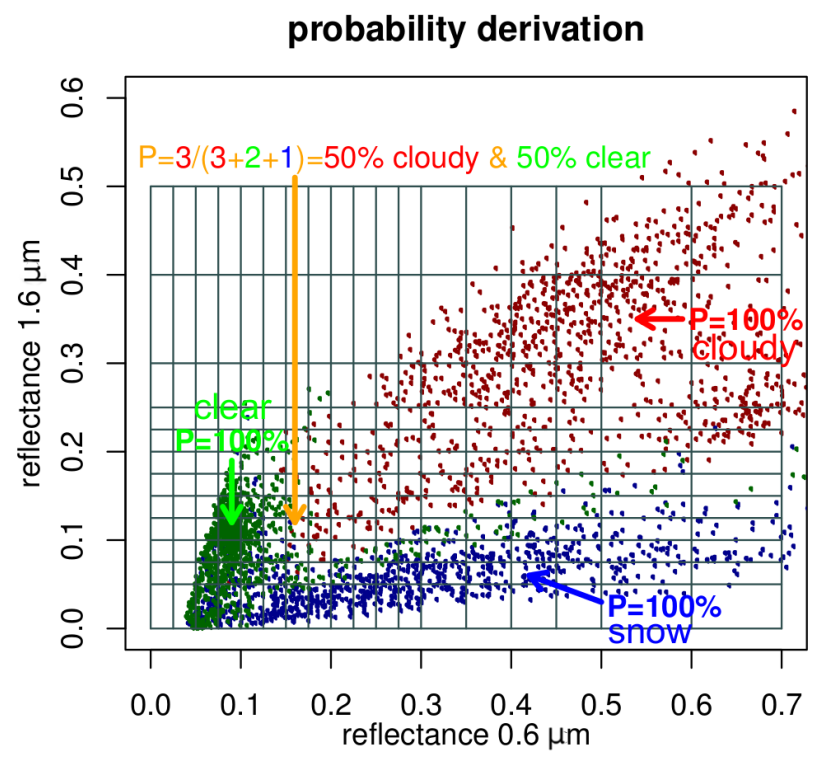

Fig. 2. Schematic graph presenting the concept of probability derivation in the PCM algorithm based on the two-dimensional spectral space composed of the reflectances at 0.6 and $1.6 \mu \mathrm{m}$. In the equation $P$ denotes probability and numbers denote the counts of cloudy, clear and snow pixels within a bin. The samples were derived from the PPS and MOD10A1 classifications of the NOAA17 satellite scene acquired over the Alps on 1 January 2008.

were not filled by the training data set probability estimates are retrieved by the nearest-neighbour interpolation of existing values within the array. At the last stage, all information spaces containing probability estimates are compressed and stored in the NetCDF4 format as the LUTs.

4. Classification of a satellite image is a procedure very similar to the construction of temporary information spaces because it requires preparation of the same spectral and ancillary data (binned values of spectral features, angles, etc.). However, this information is furthermore directly used to retrieve probability estimates from a LUT. The bottleneck of this process is related to localisation of all input data associated with a large satellite scene within a LUT, which itself has more than 60 million values. This issue is resolved by the fast approximate near-neighbour (ANN) searching method (Arya et al., 1998) which is performed for each dimension separately.

\subsection{PCM numerical implementation}

The PCM algorithm has been implemented in the R statistical language (R-project team, 2012), and the source code with help files and test cases are available on the Web: http: //pcm.r-forge.r-project.org/. The flow chart of the method is depicted in Fig. 3. The input data sets to the PCM method 


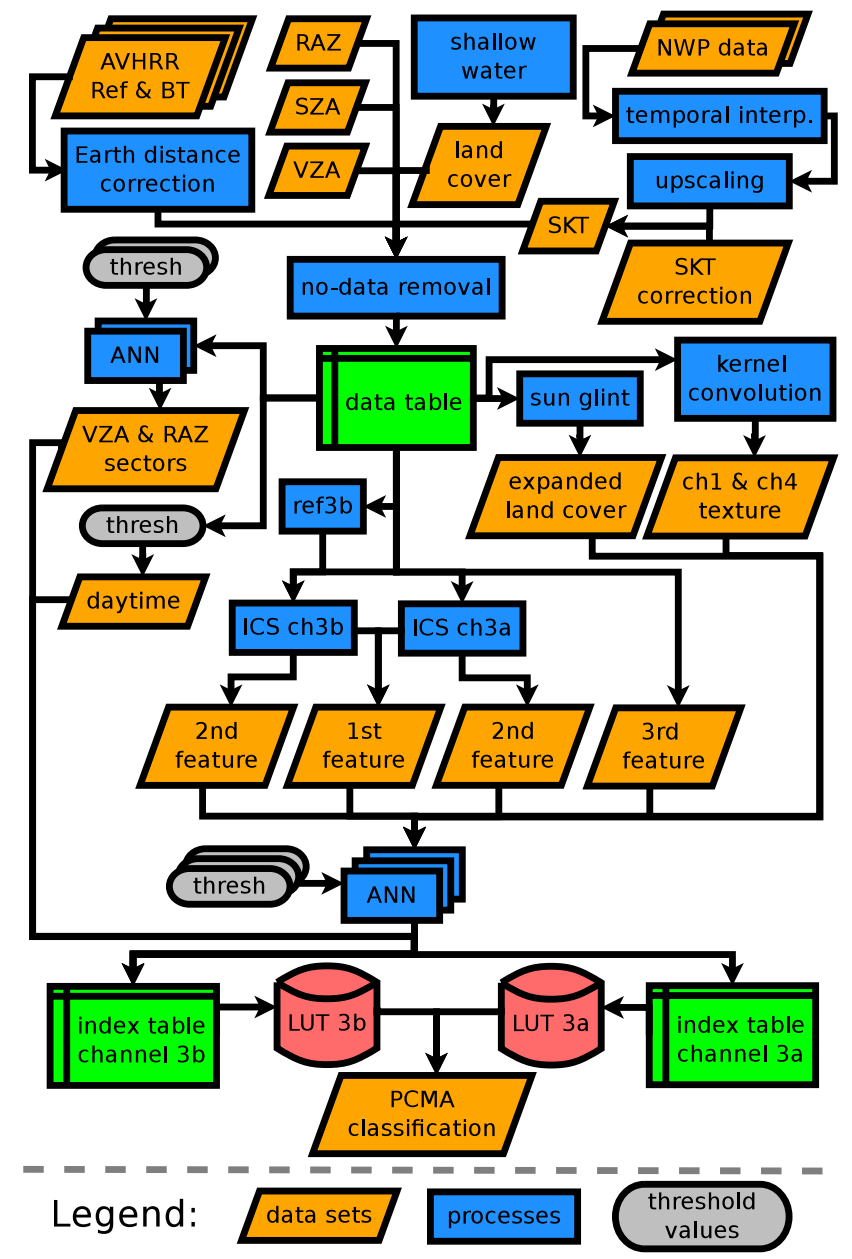

Fig. 3. Flow chart of the PCM algorithm. For details see Sect. 3.5.

require few pre-processing steps, which consist of discrimination of shallow water defined as a $8 \mathrm{~km}$ buffer zone from a shore, Earth distance correction of reflectances, and upscaling of the SKT estimates. The last process involves bilinear and temporal interpolation of two SKT estimates closest to the satellite overpass time. They are usually of much coarser spatial resolution than the AVHRR grid and correspond to certain hours. The upscaled data do not resolve well the temperature variation in rough topography terrains such as high mountains. To account for this effect, a constant temperature correction is derived by multiplying the lapse rate

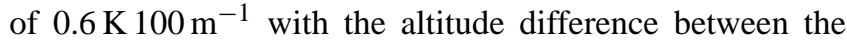
$1 \mathrm{~km} \times 1 \mathrm{~km}$ DEM provided by the USGS and the bi-linearly upscaled $0.2^{\circ} \times 0.2^{\circ} \mathrm{DEM}$ (geopotential at surface) originating from the ECMWF model. This correction is added to the final SKT estimates.

At the first stage of the PCM algorithm the required data - AVHRR reflectances and brightness temperatures, view zenith angle (VZA), SZA, RAZ, land cover, upscaled SKT and the DEM - are ingested, and no-data values are removed. Further, several processes are performed:
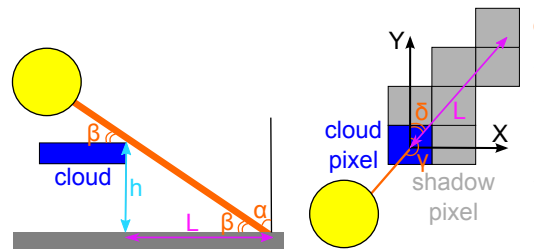

$\alpha$ sun zenith angle

$\beta$ sun elevation angle

sun azimuth angle

$\delta$ reduced azimuth angle

cloud shadow length

cloud top height

$X$ displacement along $x$ axis

$Y$ displacement along y axis

Fig. 4. Derivation of cloud shadow. For details see Sect. 3.6.

- Division between day, twilight and night (see Sect. 3.3).

- Derivation of reflectance from the $3.7 \mu \mathrm{m}$ BT.

- Identification of Sun glint areas (after Ackerman et al., 1998) over water and desert which are incorporated into the land cover data.

- Kernel convolution and derivation of the texture feature from the 0.6 and $10.8 \mu \mathrm{m}$ channels.

Further reflectances and the thermal difference between SKT and the $10.8 \mu \mathrm{m}$ BT are fetched into the ICS transformation separately suited for the $1.6 / 3.7 \mu \mathrm{m}$ channels. For the daytime data its results consist of two enhanced spectral features, where the first one together with the 10.8-12.0 $\mu \mathrm{m}$ BTD are the same for both channel configurations. For the nighttime the first two features contain scaled SKT-10.8 $\mu \mathrm{m}$ and 3.7-10.8 $\mu \mathrm{m}$ BTD respectively. Such processed spectral information, together with the texture feature, acquisition angles and expanded land cover data, are located within the binning values (see Sect. 3.3) by means of the ANN procedure (Arya et al., 1998). This leads to determination of indices along each of the eight dimensions of the information space which are merged into two separate tables depending on the $3 \mathrm{a} / 3 \mathrm{~b}$ channel availability. Finally, the index tables are used to retrieve classification probability estimates from the LUTs for every pixel in a satellite scene.

$\mathrm{SAZ}_{\text {red }}= \begin{cases}\mathrm{SAZ} \quad \in 0 \leq \mathrm{SAZ} \leq 90 ; \quad X_{\text {scale }}=-1 ; Y_{\text {scale }}=-1 \\ \mathrm{SAZ}-90 \in 90<\mathrm{SAZ} \leq 180 ; \quad X_{\text {scale }}=-1 ; Y_{\text {scale }}=1 \\ \mathrm{SAZ}-180 \in 180<\mathrm{SAZ} \leq 270 ; X_{\text {scale }}=1 ; \quad Y_{\text {scale }}=1 \\ \mathrm{SAZ}-270 \in 270<\mathrm{SAZ} \leq 360 ; X_{\text {scale }}=1 ; \quad Y_{\text {scale }}=-1\end{cases}$

$x_{2}=L \times \sin \left(\mathrm{SAZ}_{\mathrm{red}}\right) \times X_{\text {scale }}+x_{1}$

$y_{2}=L \times \cos (\mathrm{SAZ}$ red $) \times Y_{\text {scale }}+y_{1}$

\subsection{Post-classification and cloud shadow estimation}

The majority of geophysical analyses require a binary cloud and/or snow classification; thus the continuous PCM probability estimates are recoded to discrete classes assuming clear-sky pixels for the value ranges from 0 to $<50$ and from $>250$ to 300 ; cloudy pixels for the $>150$ to $\leq 250$ range and snow pixels from $\geq 50$ to $\leq 150$. Complementary to the acquired results, a cloud shadow mask is computed on the basis of simple geometrical relationships (Fig. 4). Its main 

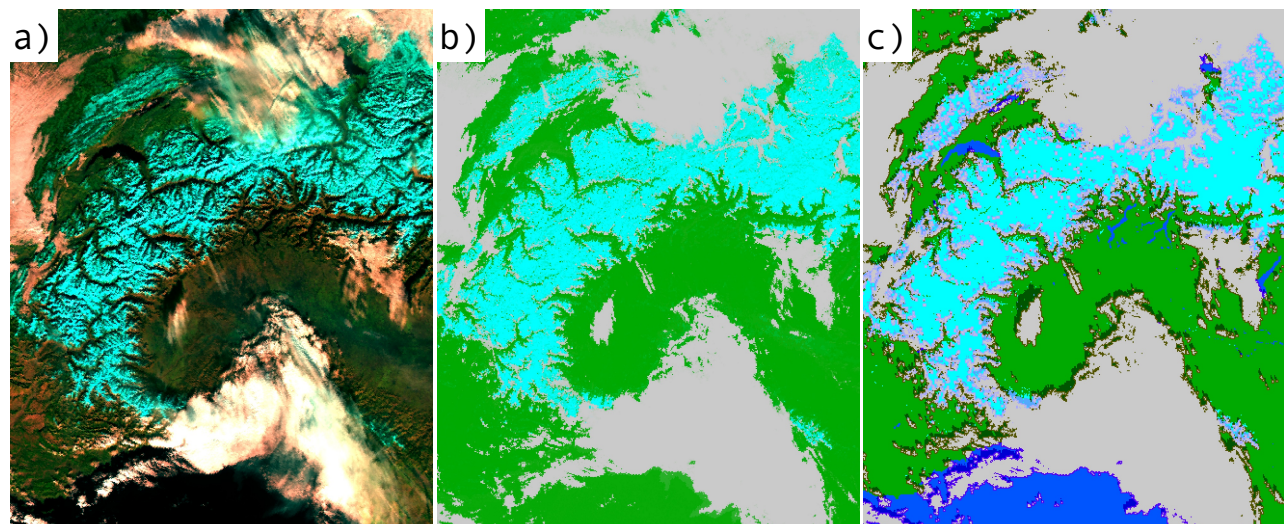

Fig. 5. PCM classification example of the NOAA17 scene acquired over the Alps on 1 January 2008 at 10:00 UTC. (a) False-colour composite $(R=1.6 \mu \mathrm{m}, G=0.8 \mu \mathrm{m}, B=0.6 \mu \mathrm{m})$, (b) probabilistic cloud and snow mask, (c) binary cloud/cloud shadow/snow/land-water mask with classes described in Sect. 3.6. In (b) and (c) grey colour depicts clouds, green depicts snow-free areas, blue depicts water and light blue depicts snow.

aim is to provide a rough approximation of a cloud shadow location which could be useful for derivation of clear-sky composites. However, if more accurate cloud shadow mask is required, please refer to the study of Simpson and Stitt (1998). In the PCM cloud shadow derivation consists of several steps. First, a rough approximation of cloud height $h$ is estimated from the SKT $-10.8 \mu \mathrm{m}$ thermal contrast assuming

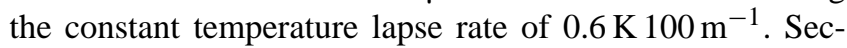
ond, the acquired altitude values are processed by the maximum value filter with a $5 \times 5$ window to enhance reliability of the estimates at cloud edges, where the thermal contrast is smaller due to fractional cloud cover. Subsequent computations are performed only for pixels located at cloud edges and initially involve calculation of shadow length $L$, expressed as cloud height divided by the tangent of Sun elevation angle $\left(90^{\circ}-\mathrm{SZA}\right)$. Next, the Sun Azimuth angle (SAZ) is reduced to $0-90^{\circ}$ range $\left(\mathrm{SAZ}_{\text {red }}\right)$, and the $X_{\text {scale }}$ and $Y_{\text {scale }}$ are derived according to the convention described by the Eq. (3). Finally, the shadow end $\left(x_{2}, y_{2}\right)$ is estimated from the coordinates of the centre of a cloud edge pixel $\left(x_{1}, y_{1}\right)$ and displacement vectors along $x$ and $y$ axis, computed as a ratio of the shadow length and sine and cosine of the $\mathrm{SAZ}_{\text {red }}$ respectively (Eq. 4).

The acquired cloud shadow mask is incorporated together with the land cover data into the PCM binary output to compose a discrete classification which consists of the following categories: no data, clear-sky water, clear-sky land, clear-sky snow, pixel adjacent to cloud over water, pixel adjacent to cloud over land, pixel adjacent to cloud over snow, cloud shadow over water, cloud shadow over land, cloud shadow over snow, and cloud. An example of the PCM output (Fig. 5) presents the NOAA17 AVHRR scene acquired over the Alps on 1 January 2008 together with the probability estimates and the discrete classification mask.

\subsection{Limitations of the PCM algorithm}

Apart from the advantages of the PCM method - such as determination of classification probability for clear-sky, snow and cloudy conditions, and unsupervised algorithm training phase - there are some methodological limitations. Although the algorithm provides a great flexibility over other classification methods in terms of selection of numerous binning values, their choice is still a matter of subjective decision. Nevertheless, it is possible to determine an arbitrary number of regularly distributed binning values for each feature, and still the algorithm will exhibit considerable detection skills. The approach applied in this study assumes higher density of bins around value ranges associated with uncertain pixels (e.g. low reflectance values related to broken/cirrus clouds). Furthermore, the number of intervals modifies the probability values, as wider bins within the information space are more likely to contain a mixture of classes in comparison to smaller bins. Thus, a high density of binning values assures better classification accuracy at the price of bigger LUT size, which itself is a limiting factor.

The quality of the PCM results mostly depends on the accuracy of the clear-sky/snow/cloud mask used during the algorithm training step. Moreover, the quantity of training data should be large enough to sufficiently sample the multidimensional array with information acquired under a wide range of environmental conditions. Although in this study a broad set of ecosystems was considered (from deserts to tundra and perennial ice), the performance of the method over other regions than Europe is still to be determined, as well as the applicability to the older AVHRR sensor (prior to NOAA16). 


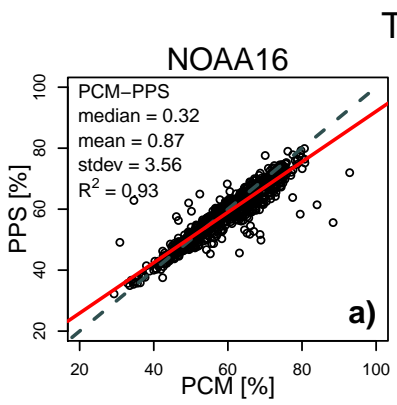

Total cloud cover PCM vs PPS
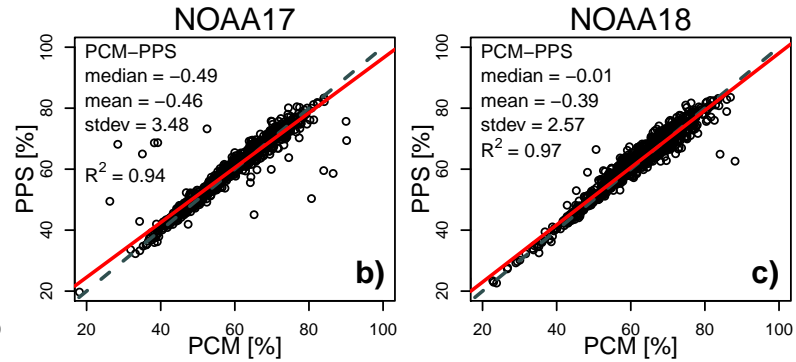

Fig. 6. Scatter plots of the total cloud cover estimates computed over each of the NOAA16, 17 and 18 satellites scenes separately for the PCM and PPS algorithms. Red line denotes linear trend between these two data sets. Some statistics of the PCM-PPS total cloud cover differences distribution are reported. For details see Sect. 4.1.

\section{PCM cloud and snow classification results}

The accuracy assessment of the PCM algorithm was divided into four parts, where first the results were compared to the original PPS cloud mask version 2012 in order to verify agreement between training data set and the acquired output. Then, the MODIS cloud mask (MOD/MYD35 collection 6 products) and daily snow cover composite (MOD10A1 collection 5 product) were used as a reference to compare the PCM results with other robust classification algorithms suited for a modern sensor with higher radiometric resolution than AVHRR. Further, the PCM output was validated against ground-truth data originating from more than 2000 manually operated SYNOP stations which provide detailed cloudiness observations. Finally, it was validated against the vertical feature mask version 3 derived from the CALIOP (CloudAerosol Lidar with Orthogonal Polarization) lidar measurements. All of the mentioned data sets feature a discrete form; therefore continuous PCM probability estimates, coded according to the convention described in Sect. 3.4, were transformed to a binary form. This was achieved assuming the clear-sky pixels for the coded probability values of $0-50$ and 250-300; cloudy pixels for the 150-250 range and snow pixels for the 50-150 range.

\subsection{Intercomparison with the PPS cloud mask}

In order to evaluate discrepancies between the PCM and PPS algorithms the total cloud cover estimates expressed as $100 \% \times \frac{\text { cloudy pixels }}{\text { total pixel count }}$ over each satellite scene were computed. The acquired results for both methods were linearly regressed and basic statistics of a distribution of differences were derived (Fig. 6). It was found that both methods are in good agreement regardless of the image acquisition time, being morning satellites NOAA 16 and 17 and the afternoon one NOAA18, as well as the channel configuration: $1.6 \mu \mathrm{m}$ for NOAA17 and $3.7 \mu \mathrm{m}$ for NOAA16,18. The total cloud cover differences between PCM and PPS outputs are low: $-0.5 \%$ to $1.0 \%$ on average, with standard deviations not exceeding $3.6 \%$ and with correlations $\geq 0.93$.
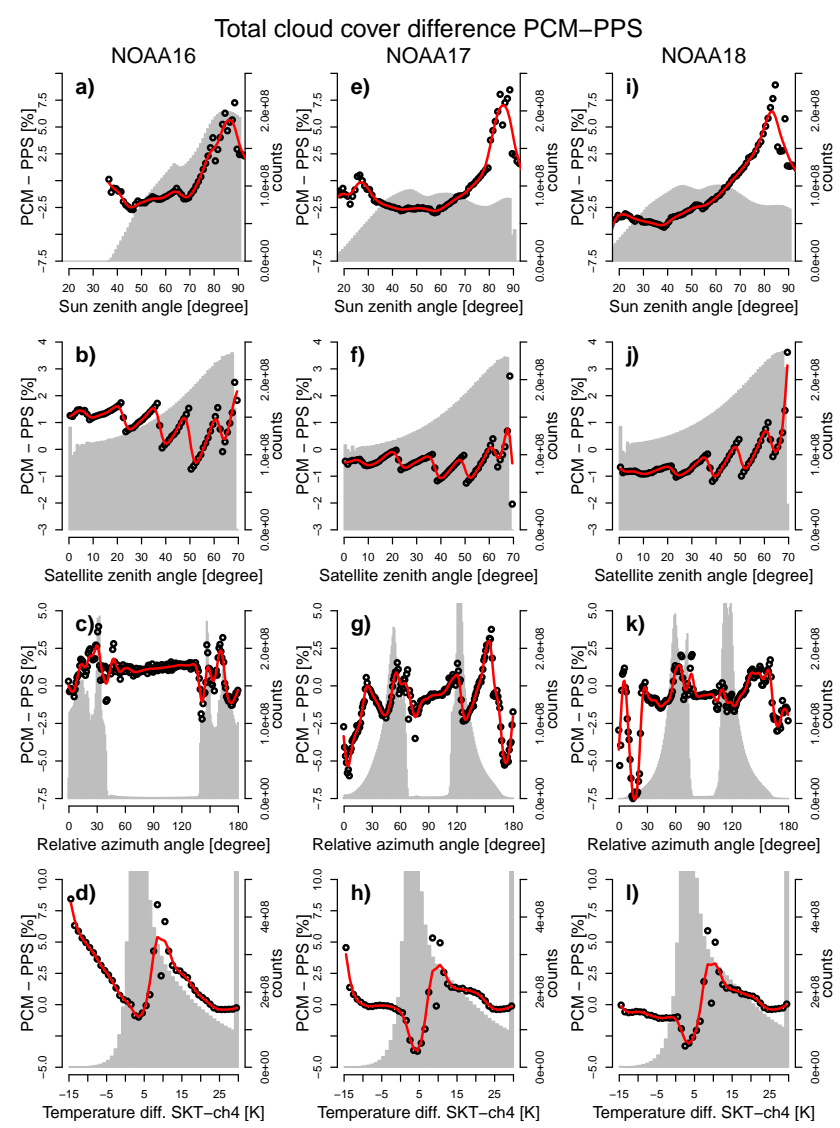

Fig. 7. Total cloud cover differences PCM-PPS as a function of selected variables derived from the annual pixel counts for the years 2011 (NOAA16) and 2008 (NOAA17 and 18). Data frequency is presented as grey-shaded histograms. Red lines denote trends computed by the smoothing spline method. For details see Sect. 4.1.

Further, relationships between the PCM-PPS differences and angular/thermal conditions (Fig. 7) and land cover (Fig. 8) were investigated. This involved derivation of annual histograms for the selected variables (grey shading in Fig. 7) and computation of total cloud cover within each bin. 


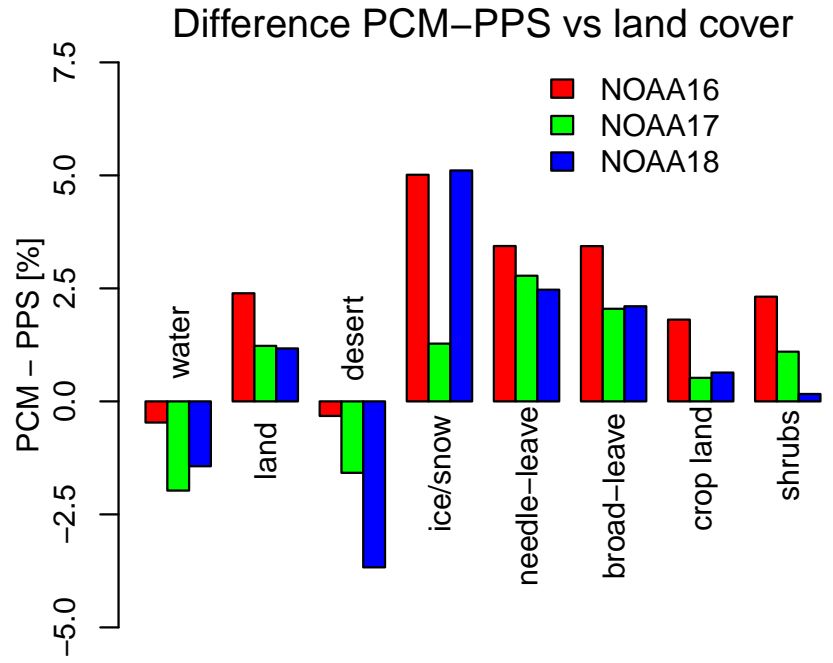

Fig. 8. Total cloud cover differences PCM-PPS as a function of land cover derived from the annual pixel counts for the years 2011 (NOAA16) and 2008 (NOAA17 and 18). For details see Sect. 4.1.

As far as the Sun zenith angle is concerned, the PCM reports more clouds for higher angles than PPS. This relation starts to decrease again above the angle of $85^{\circ}$ which determines the twilight conditions. For the satellite zenith angle, the PCM-PPS difference distribution features a characteristic "saw-shape" pattern which originates from the dependency of probability estimates on the angular sectors. The variation of discrepancies increases towards oblique angles, but the values stay within -2 to $2 \%$ range. Contrarily, the relative azimuth angle between the Sun and a satellite does not influence the difference in a systematic way. It seems that the majority of the variation can be associated with the number of observations acquired at specific angular conditions. The relationship between the PCM-PPS total cloud cover differences and the thermal contrast between SKT and the $10.8 \mu \mathrm{m}$ channel features a distinct pattern. For negative values (SKT lower than $10.8 \mu \mathrm{m}$ ), associated with a temperature inversion or with an underestimated SKT prediction, the PCM method tends to report more clouds than PPS (up to $8 \%$ ). This situation often occurs during the night over the sparsely vegetated areas (Sahara, Spain), where climate models poorly represent rapid radiative cooling, which leads to erroneous temperature inversions. This changes the PPS parametrisation and probably results in lower total cloud cover (Fig. 10). For small positive thermal contrast $0-5 \mathrm{~K}$ PPS reports more clouds, but just beyond this range the relation changes rapidly and around $10 \mathrm{~K}$ there is a positive peak, where PCM detects more clouds. Above this value the total cloud cover differences get smaller, as high thermal contrast between the surface and satellite measurements is usually associated with the overcast conditions. Considering the land cover classes (Fig. 8), the PPS method reports more clouds over water bodies (up to $2 \%$ ) and desert (up to $5 \%$ ), while
PCM detects more clouds over land (up to $2.5 \%$ ), especially over perennial snow/ice (up to $5 \%$ ) but only for satellites without the $1.6 \mu \mathrm{m}$ channel activated. Over vegetated areas, the differences are notably pronounced (up to $4 \%$ ) for the needle-leaved forest class, which covers high-latitude zones and mountainous regions. For the broad-leaved forest the discrepancies are slightly lower; however they are still 1 to $1.5 \%$ higher than for croplands or shrubs. This may indicate that the PCM method is more sensitive to cloud cover over dark dense vegetation in comparison to PPS.

The next step of the investigation involved spatial analyses of the annual total cloud cover composites over Europe separately performed for day and nighttime conditions. Its results (Figs. 9 and 10) for the PPS data reveal areas with unreliably high cloud coverage over desert and Spain, which are significantly less pronounced on the PCM composition. These areas are related to land cover categories distinguished by the USGS classification (Anderson, 1976) and utilised by the PPS which do not reflect well the local spectral properties of the surface. On the other hand, PCM takes as input more detailed GLC2000 classification (Bartholomé and Belward, 2005) where classes are more spectrally uniform, which does not lead to cloud overestimation. Another problematic region is associated with mountains where PPS reports less clouds especially during the nighttime due to the cloud conservative threshold $(-22 \mathrm{~K})$ in the $10.8 \mu \mathrm{m}-\mathrm{SKT}$ test. This effect is particularly noticeable over Norway and the Alps. Nevertheless, most of the spatial cloud coverage patterns visible on the PPS image are also present in the PCM composite, which confirms a good agreement between these methods.

Finally, the spatio-temporal aspect of the PCM-PPS total cloud cover discrepancies was analysed as a function of latitude and time (Fig. 11). For all of the selected satellites and both years 2008 and 2011 it could be seen that the temporal pattern of differences does not change over the coarse of the year. In the comparison to the PPS method, PCM tends to detect more clouds for the latitudes from 30 to $40^{\circ}$ and 50 to $60^{\circ}$, while for the latitudes from 40 to $50^{\circ}$ and 60 to $70^{\circ}$ it reports less of them. This pattern corresponds to the spatial distribution of land and water over Europe, where PCM reports more clouds over land, whereas PPS reports more of them over water (Fig. 8).

\subsection{Intercomparison with MODIS cloud and snow mask}

The PCM classification consists of the cloud and snow components; thus to assess its accuracy two types of products derived from the MODIS data were used as a reference. The first one - MOD35/MYD35 - consists of a cloud mask (Ackerman et al., 1998) generated from a 5 min swath segment recorded by the TERRA and AQUA satellites. In order to compare the cloud masks derived from the MODIS and AVHRR data, image acquisition times had to be collocated, as the cloud cover dynamics requires a small interval 
N18 PCM total cloud cover during day in 2008

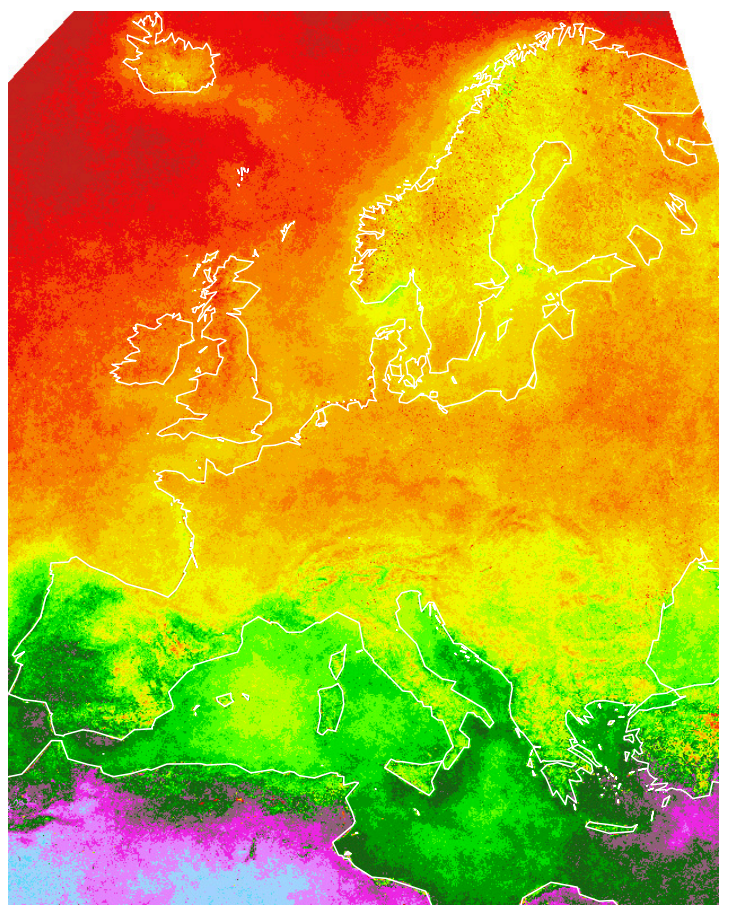

N18 PPS total cloud cover during day in 2008

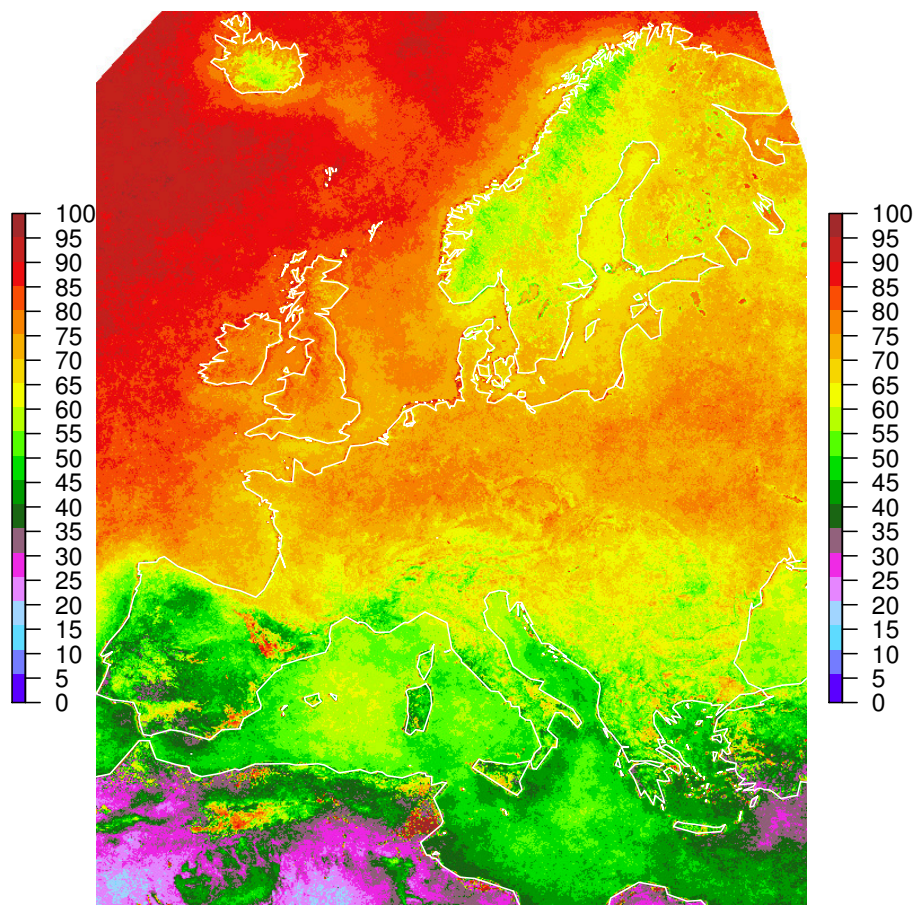

Fig. 9. PCM and PPS annual total cloud cover composites in 2008 derived from the collection of daytime NOAA18 scenes.

N18 PCM total cloud cover during night in 2008

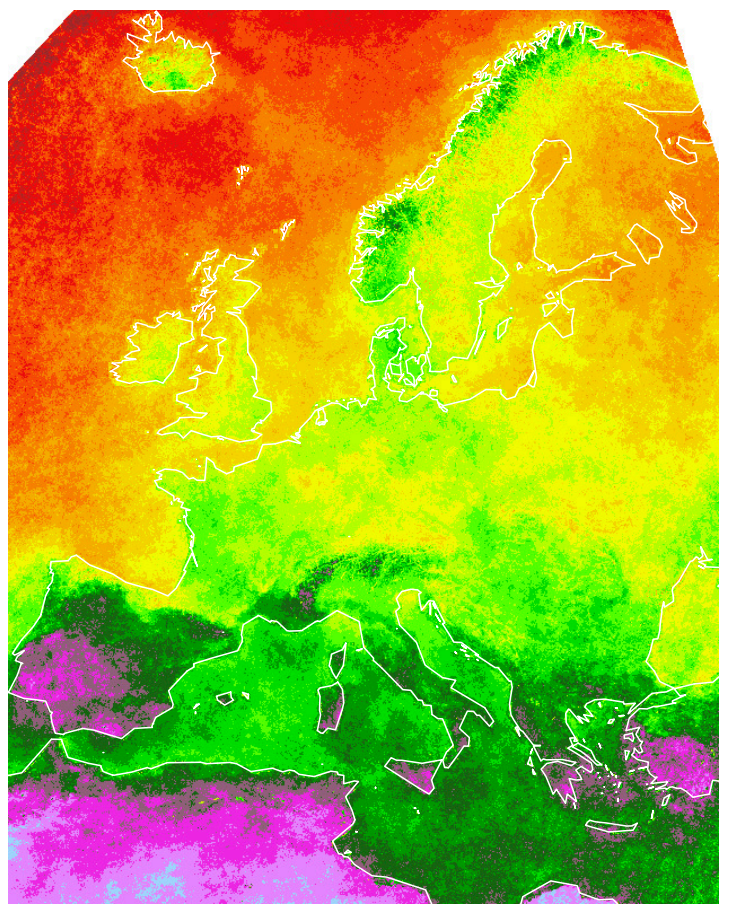

N18 PPS total cloud cover during night in 2008

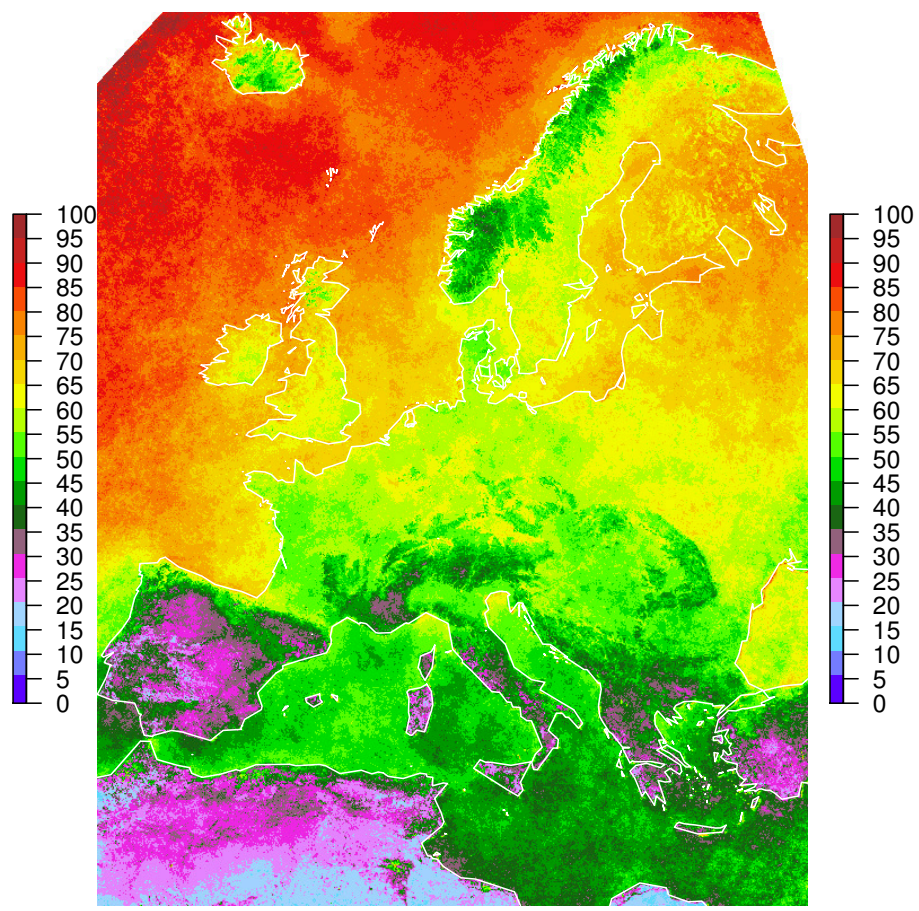

Fig. 10. PCM and PPS annual total cloud cover composites in 2008 derived from the collection of nighttime NOAA18 scenes. 
a) NOAA16 PCM-PPS total cloud cover in 2011

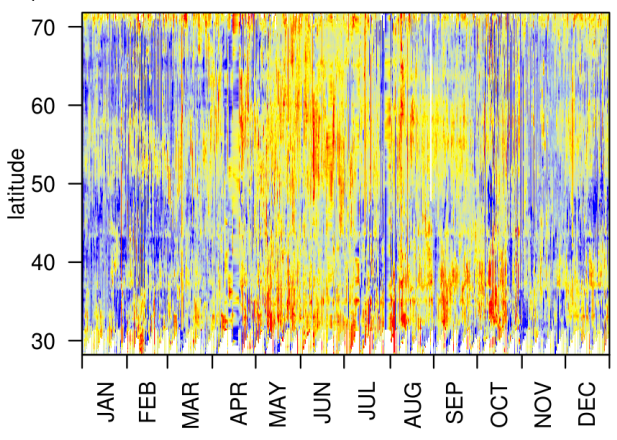

b) NOAA17 PCM-PPS total cloud cover in 2008

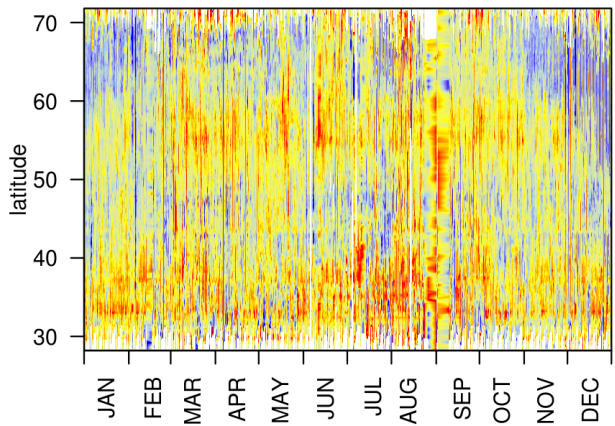

c) NOAA18 PCM-PPS total cloud cover in 2008

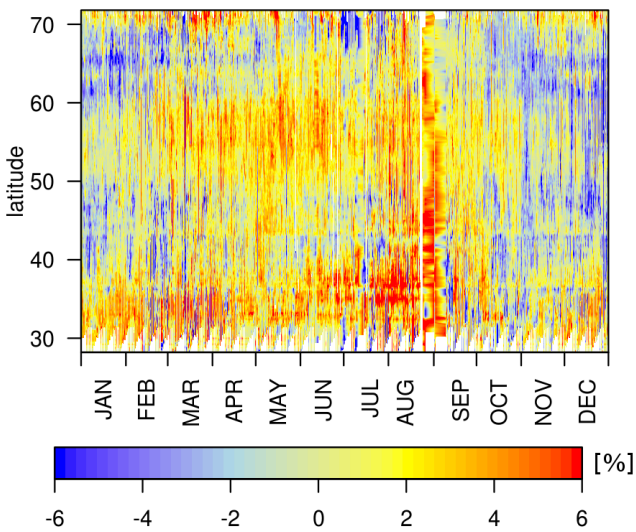

Fig. 11. Distribution of latitudinal total cloud cover differences between the PCM and PPS algorithms throughout the years 2011 and 2008 for the selected satellites. For details see Sect. 4.1.

between spectral measurements. In this study the maximum time difference was set to $15 \mathrm{~min}$. Out of the three selected NOAA platforms only the one labelled with number 18 was used for the comparison. In the case of the NOAA16 in the year 2011 the orbital drift was substantial; thus no collocations were possible with TERRA/AQUA. For the NOAA17, match-ups should occur for high latitudes; however those scenes were beyond the range of the receiving station antenna located in Bern (Switzerland). Over Europe for the year 2008 after the image collocations the combined AVHRR/MODIS data set consisted of 118 TERRA scenes and 238 AQUA scenes for both day and night conditions. Furthermore, the
MODIS product was modified to exclude the uncertain pixel category and to reclassify the confident/probably clear categories to clear-sky class, as well as the confident/probably cloudy categories to overcast class.

Regarding the MOD/MYD35 product, the comparative analyses were performed analogically to the ones described in the previous subsection. First, the total cloud cover over each scene was computed for both cloud masking algorithms to compose a scatter plot (Fig. 12a). It occurs that on average the MODIS product reports $4.4 \%$ more clouds than PCM remaining at the same time with the high correlation of 0.95 . Further, the PCM-MODIS total cloud cover discrepancies were analysed as a function of land cover, Sun zenith angle and the SKT-10.8 $\mu \mathrm{m}$ thermal contrast (Fig. 12b, c and d). The obtained results show that the PCM constantly reports less clouds regardless of the land cover class with the highest difference for the perennial snow/ice areas (up to $12 \%$ ). As far as the Sun zenith angle is concerned, the absolute differences increase almost linearly, and the best agreement between the methods $( \pm 2.5 \%)$ is reported for the $\mathrm{SZA}<55^{\circ}$. On the other hand, for the twilight conditions $\left(\mathrm{SZA} \geq 85^{\circ}\right)$ total cloud cover discrepancies between PCM and MOD35 reach $10 \%$. Their relationship with the SKT-10.8 $\mu \mathrm{m}$ thermal contrast (Fig. 12d) resembles the one from the PPS comparison. When the surface temperature is lower than satellite measurements, PCM reports more clouds (up to $5 \%$ ). For the thermal difference in the 0 to $5 \mathrm{~K}$ range the PCM-MODIS absolute discrepancies increase (up to $18 \%$ ) and then diminish rapidly, and above the $10 \mathrm{~K}$ both algorithms report the same amount of clouds.

The MOD10A1 product (Hall et al., 2002) utilised as a reference for the PCM classification consists of daily snow cover composites retrieved from the TERRA satellite data. As it is one of the few globally available snow cover data with high spatial and temporal resolutions, in this study it was regarded as ground-truth information. On the basis of the MOD10A1 and PCM products the contingency table was constructed (Eq. 5), which allows computation of the following classification quality indicators (Eqs. 6-11): probability of detection (POD), false alarm rate (FAR), hit rate (HR), and Kuipers skill score (KSS). The acquired results indicate that the difference in estimated snow cover between the PCM and MOD10A algorithms is significant (Fig. 14). Considering the $\mathrm{PCM}$ snow detection skills, the highest, $\mathrm{POD} \approx 0.6$, is reported for the NOAA17 which has the $1.6 \mu \mathrm{m}$ channel activated. On the other hand, the AVHRR sensors aboard the NOAA16,18 satellites with the $3.7 \mu \mathrm{m}$ channel constantly operating feature lower snow detection skills: $\mathrm{POD} \approx 0.4$. The prevailing snow-free conditions over the coarse of the year result in extremely high POD, HR and low FAR for this category, due to unbalanced data counts within the contingency table. In such a case, the KSS indicator should be utilised, as it objectively estimates the overall algorithm performance. Further, the total snow cover scatter plots, derived analogically to the ones from the cloud coverage analyses, were 

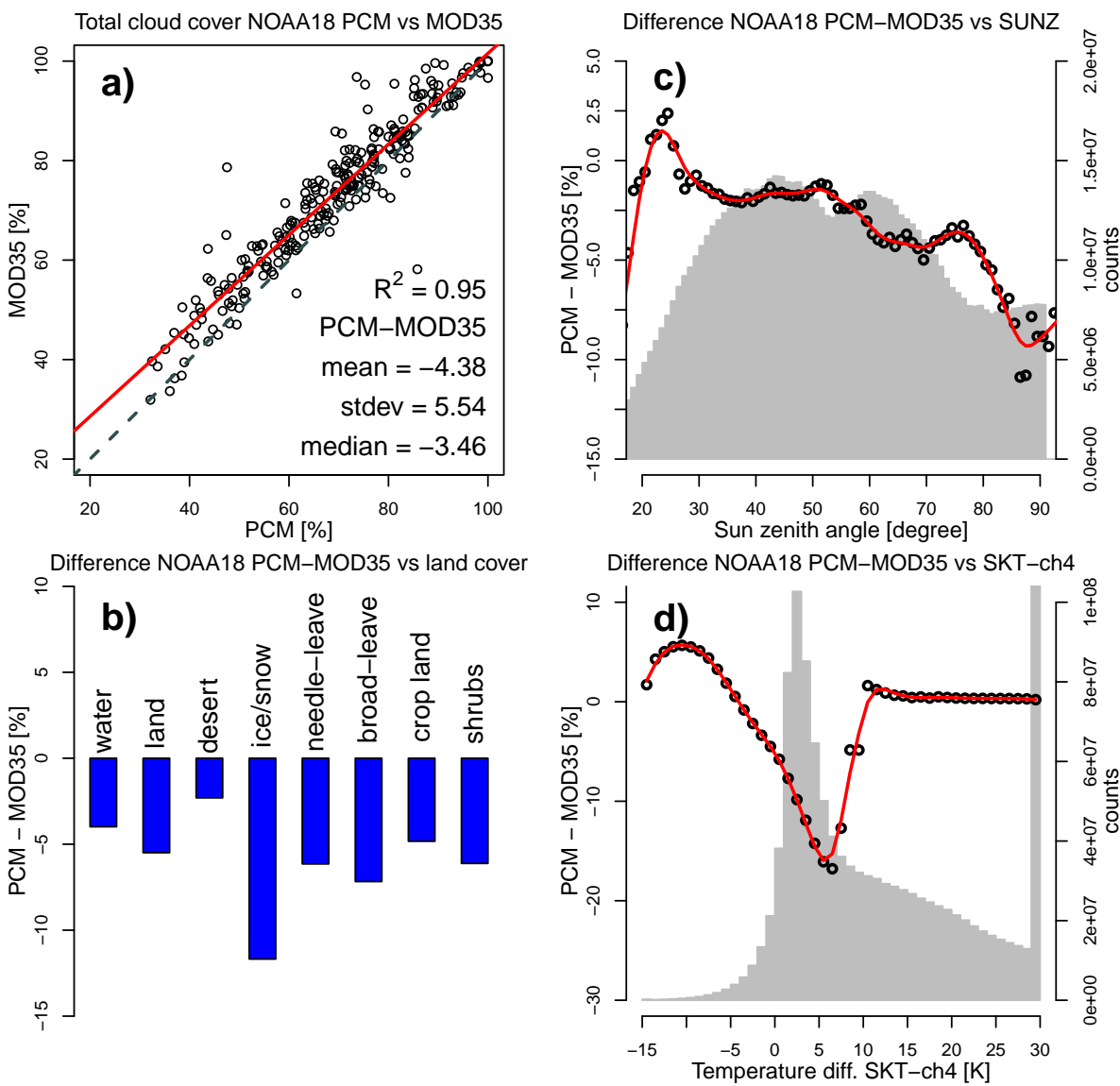

Fig. 12. Differences in total cloud cover between PCM and MOD/MYD35 products derived from annual pixel counts of the NOAA18 satellite for the year 2008: (a) computed over each scene, (b) as a function of land cover, (c) as a function of Sun zenith angle, (d) as a function of thermal differences between SKT and $10.8 \mu \mathrm{m}$ channel. Red lines denote trend, and data frequency is presented as grey-shaded histograms. For details see Sect. 4.2.

computed (Fig. 13d, h and l). The obtained results show that PCM reports significantly less snow cover (up to $9.5 \%$ on average) than MOD10A1, with a moderate correlation coefficient (0.5-0.7) and a high standard deviation (up to $17 \%$ ). The absolute PCM-MOD10A1 total snow cover differences increase with the Sun zenith angle up to $85^{\circ}$, where they slightly decrease due to separate treatment of the twilight conditions (Fig. 13a, e and i). The relationship with the satellite zenith angle indicates that PCM reports more snow at high angles, possibly due to increasing instrument field of view (FOV), but only for the NOAA16 and 17 platforms. The opposite trend for the NOAA18 satellite is not easily explainable and might be related to geographic distribution of snow in Europe, which is most persistent over the Alps and Scandinavia. These regions are located around the centre of a swath of the NOAA18 satellite; thus the amount of snow pixels across the scan is not even. The PCM-MOD10A1 total snow cover differences vary with the relative azimuth angle according to a pattern mostly related to the quantity of available observations. Nevertheless, for the NOAA18 there is a significant increase of discrepancies around the azimuth angle of $60^{\circ}$ (towards forward scattering), which once more might be associated with combined geographical and angular conditions rather than the algorithm's configuration.

$$
\begin{aligned}
& \text { counts PCM clear PCM snow } \\
& \text { MODIS clear } a \quad b \\
& \text { MODIS snow } c \quad c \quad d \\
& \mathrm{POD}_{\text {snow }}=\frac{d}{c+d} \\
& \mathrm{POD}_{\text {clear }}=\frac{a}{a+b} \\
& \text { FAR }_{\text {snow }}=\frac{b}{b+d} \\
& \text { FAR }_{\text {clear }}=\frac{c}{a+c} \\
& \mathrm{HR}=\frac{a+d}{a+b+c+d}, \text { where } 0 \leq \mathrm{HR} \leq 1 \\
& \mathrm{KSS}=\frac{a \times d-c \times b}{(a+b) \times(c+d)}, \text { where }-1 \leq \mathrm{KSS} \leq 1
\end{aligned}
$$



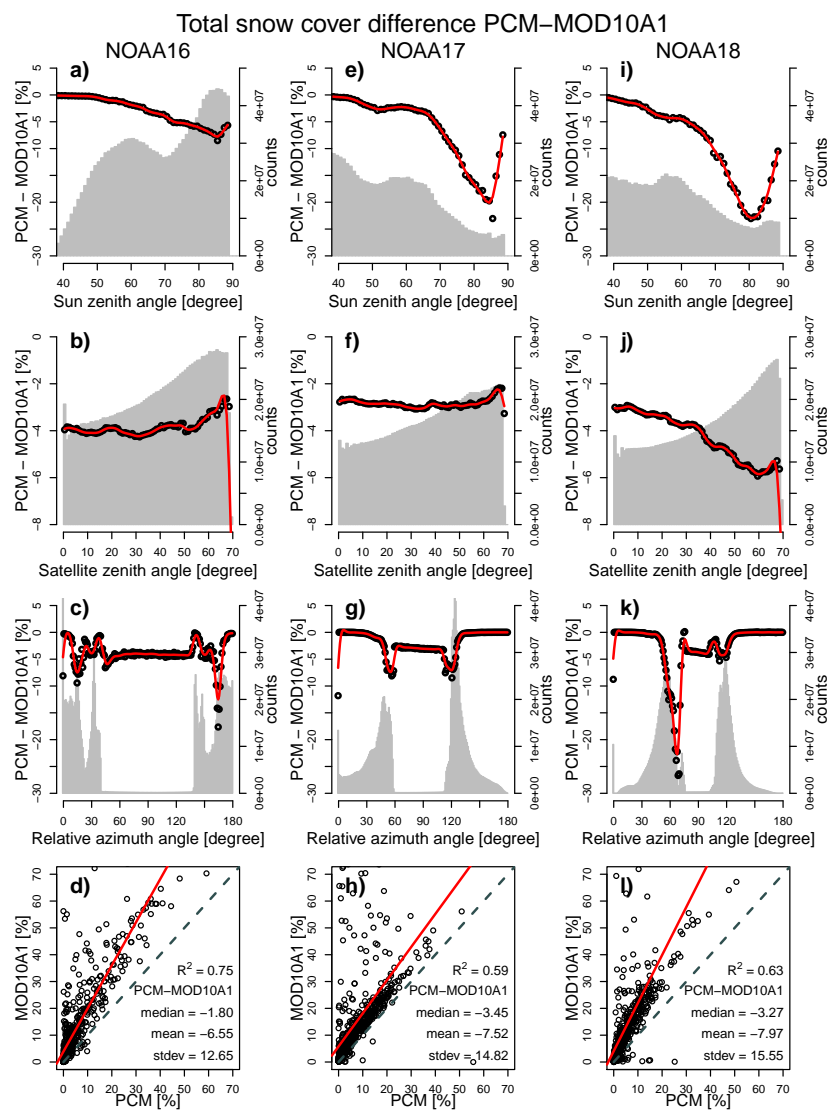

Fig. 13. Total snow cover difference PCM-MOD10A1 as a function of selected variables derived from annual pixel counts for the years 2011 (NOAA16) and 2008 (NOAA17 and 18). Data frequency is presented as grey-shaded histograms. Bottom panels present the total snow cover scatter plots derived from estimates computed over each scene. Red lines denote trends. For details see Sect. 4.2.

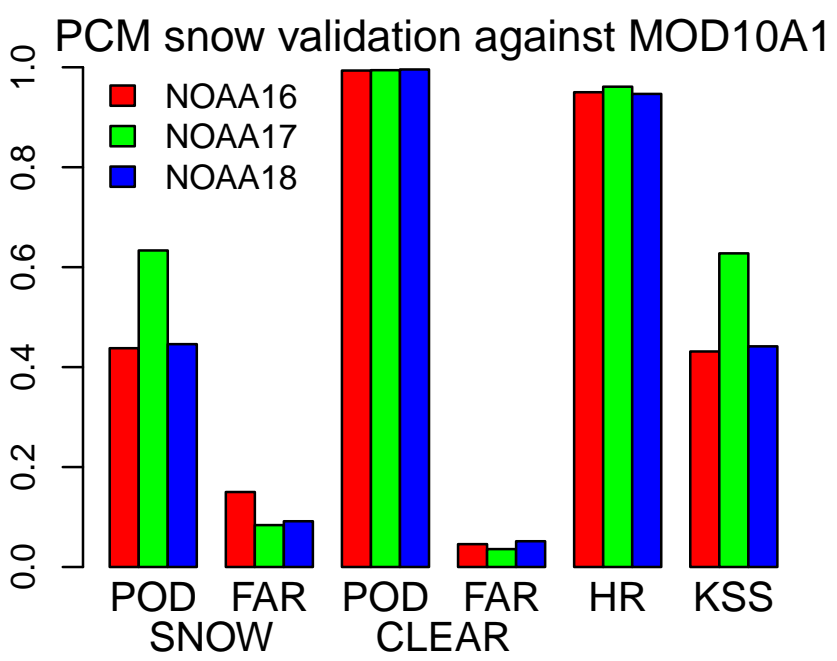

Fig. 14. Accuracy indicators of the PCM snow detection skills derived from the comparison with the MOD10A1 product. For details see Sect. 4.2.

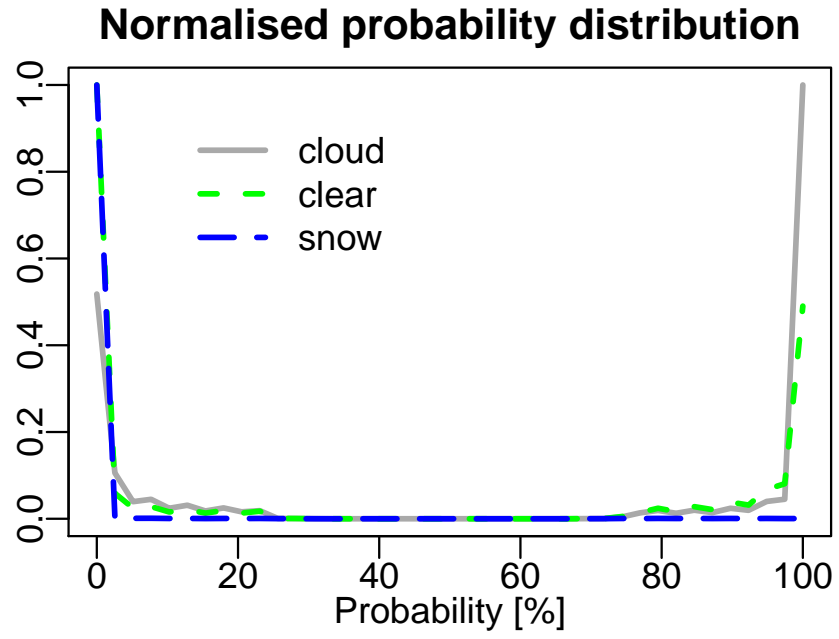

Fig. 15. PCM normalised probability distribution for clear, cloudy and snow conditions derived from annual counts of the NOAA18 satellite data. For details see Sect. 4.3.

\subsection{Validation against SYNOP weather reports}

The global network of SYNOP stations (Fig. 16a) provides detailed cloud cover observations acquired at high temporal resolution (up to one hour). They are collected in a consistent manner during day and night for a long period, which makes them an excellent validation source for the satellite-based products. In this study SYNOP total cloud cover observations expressed in octants were compared to the PCM cloud probability, which was recoded to $0-100 \%$ range, where $0 \%$ denotes confident clear/snow class and $100 \%$ indicates confident cloudy category. In order to maintain the coherence between these data sets, the PCM outputs in a binary form (clear/cloudy) and the cloud probability were averaged within the $30 \mathrm{~km}$ buffer zone around each station. This area should correspond to the sky extent usually visible to an observer at the station (Karlsson, 1995). Analogically to the comparison with the MOD/MYD35 data, the satellite and ground cloud observations were collocated within the $15 \mathrm{~min}$ interval. Next, the mean PCM cloud probability was computed as functions of the SYNOP cloud amount and time of day from all of the collocated stations and satellites in the selected years (Fig. 16b-d). The acquired probability distributions span from around $12 \%$ for completely clear-sky SYNOP observations to $90 \%$ for fully overcast conditions regardless of the time of day. Furthermore, the histograms of annual normalised probability distributions for clear, cloudy and snow conditions were computed (Fig. 15). Their shape very well corresponds to other probabilistic cloud mask distributions enclosed in the studies of Gómez-Chova et al. (2007) and Heidinger et al. (2012).

In order to compute the quality indicators (Eqs. 5-11 with cloudy instead of snow) the PCM and PPS cloud fractions derived over the $30 \mathrm{~km}$ buffer zone together with the SYNOP 
a)

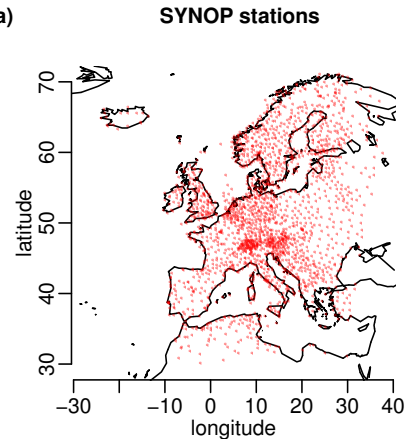

c) Mean PCM cloud probability vs SYNOP day cloudiness

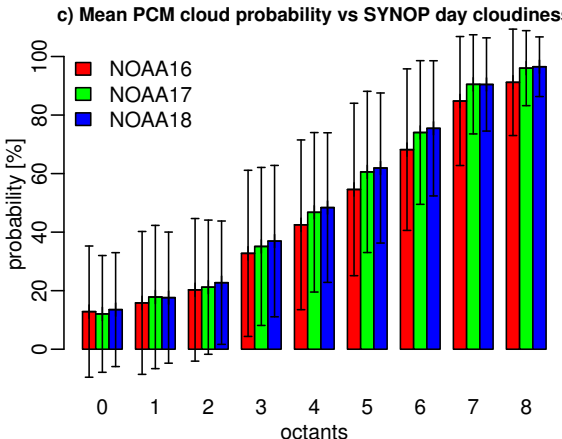

b) Mean PCM cloud probability vs SYNOP cloudiness

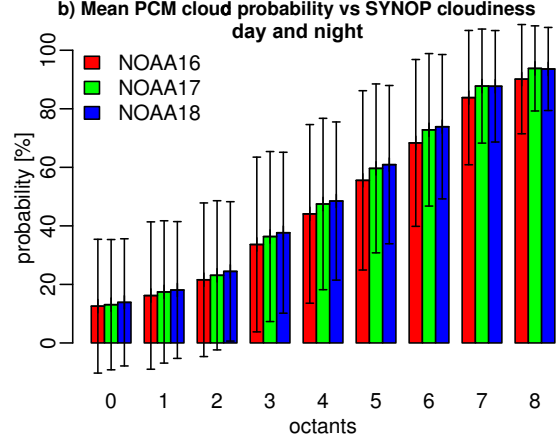

d) Mean PCM cloud probability vs SYNOP night cloudiness

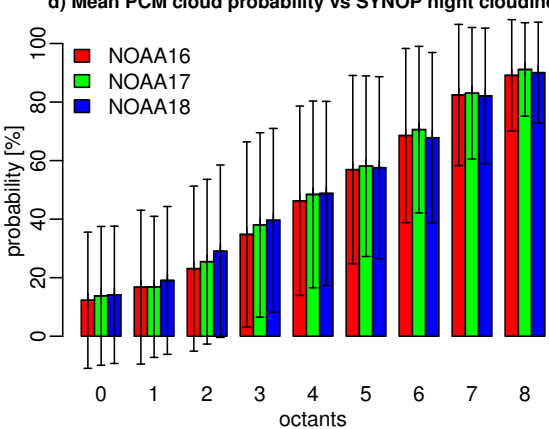

Fig. 16. (a) Geographic distribution of SYNOP stations. (b)-(d) Distribution of mean PCM cloud probability as a function of SYNOP cloud amount expressed in octants for different times of day. Error bars denote standard deviations. For details see Sect. 4.3.

observations were transformed to a binary form assuming overcast conditions over $25 \% / 2$ octants' cloud amount. The obtained results proved fine cloud detection skills of both algorithms (high POD, HR, KSS and low FAR) without any substantial differences between them. The overall accuracy during the night is a bit lower than during the day, which is to be expected provided the availability of the thermal information only. Amongst the selected satellites, the NOAA17 platform with $1.6 \mu \mathrm{m}$ configuration provides the most reliable cloud coverage data during the day, whereas for the nighttime the best results are obtained by the NOAA18 platform. Nevertheless, the differences in cloud detection skills between different satellites are small for both PCM and PPS algorithms.

\subsection{Validation against CALIPSO/CALIOP lidar feature mask}

The last part of the performed analyses involved validation of the PCM and PPS cloud masks against the CALIOP vertical feature mask (CAL_LID_L2_VFM). The CALIOP lidar is an active system which is able to detect very thin cloud layers with high horizontal resolution of $333 \mathrm{~m}$. This makes it an extremely valuable source for validation of any medium-resolution satellite cloud mask (Karlsson and Dybbroe, 2010). Nevertheless, before such a comparison is possible satellite observations have to be collocated in the space and in the time domains. In this study the approach to this issue consists of three steps. First, CALIOP observations with a time interval lower than $15 \mathrm{~min}$ as compared to the AVHRR acquisition time were selected. Next, the vertical feature mask was transformed to a vector with a binary flag present whenever a cloud layer was reported within an atmospheric column. Finally, the location of acquired samples within the $1 \mathrm{~km} \times 1 \mathrm{~km}$ AVHRR grid was determined by means of the nearest-neighbour technique. The CALIPSO satellite shares the same orbit as the AQUA platform with the MODIS instrument; thus according to the arguments given in Sect. 4.2 only a comparison with the NOAA18 satellite was possible.

From the collocated CALIOP/NOAA18 observations the quality indicators of the PCM and PPS cloud detection skills were computed. The acquired results (Fig. 18) confirmed good performance of both algorithms with slightly better indicators in the case of the PCM algorithm. The absolute values of computed quality indicators insignificantly differ from those presented in the AVHRR/SYNOP validation and the relationships between them remain the same. The POD for cloudy conditions is lower by around 0.05 for the nighttime acquisitions in comparison to the day ones, while the FAR features a reverse trend. This in turn results in higher FAR for the clear conditions during night. Nevertheless, all of those differences are small and overall PCM/PPS cloud detection accuracy expressed by the KSS indicator is almost the same regardless of the time of day. 
PCM \& PPS cloudiness vs all SYNOP observations

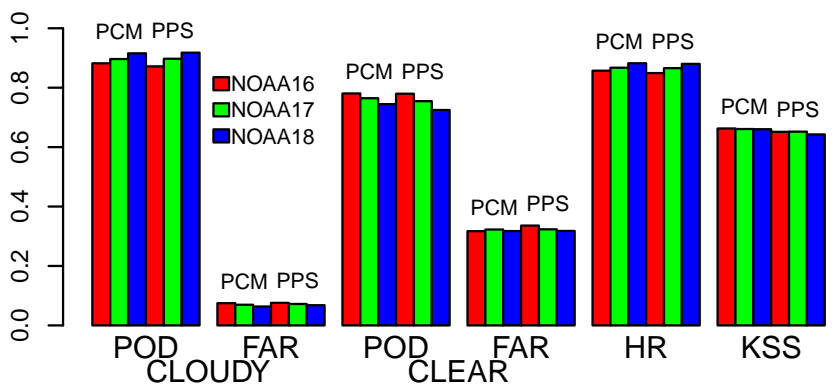

PCM \& PPS cloudiness vs SYNOP day observations

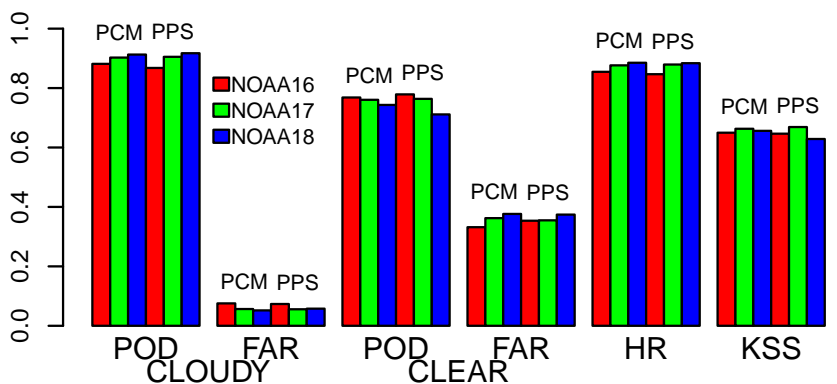

PCM \& PPS cloudiness vs SYNOP night observations

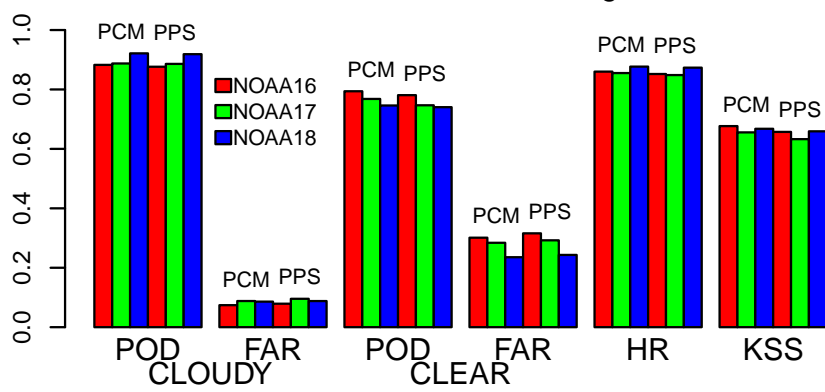

Fig. 17. Accuracy indicators of the PCM and PPS cloud detection skills derived from the comparison with SYNOP cloud observations. For details see Sect. 4.3.

\section{Discussion}

The performed analyses were designed to assess the accuracy of the PCM algorithm from the perspective of cloud and snow detection skills. The utilised reference data sets feature a discrete form; therefore the PCM probability estimates between snow-free/snow, snow/cloudy and cloudy/clear-sky conditions were transformed to binary classes using threshold values described in Sect. 4. Such a prepared data set was then compared to the PPS and MODIS cloud masks, MOD10A1 snow mask, CALIOP vertical feature mask and SYNOP total cloud amount observations. As far as the classification probability is concerned, the lack of an appropriate reference data set allows only a basic comparison of the PCM cloud probability against the SYNOP total cloud amount measurements. Moreover, computed normalised probability distribution for cloudy conditions (Fig. 15) is consistent with the findings of Gómez-Chova et al. (2007) and Heidinger et al. (2012).
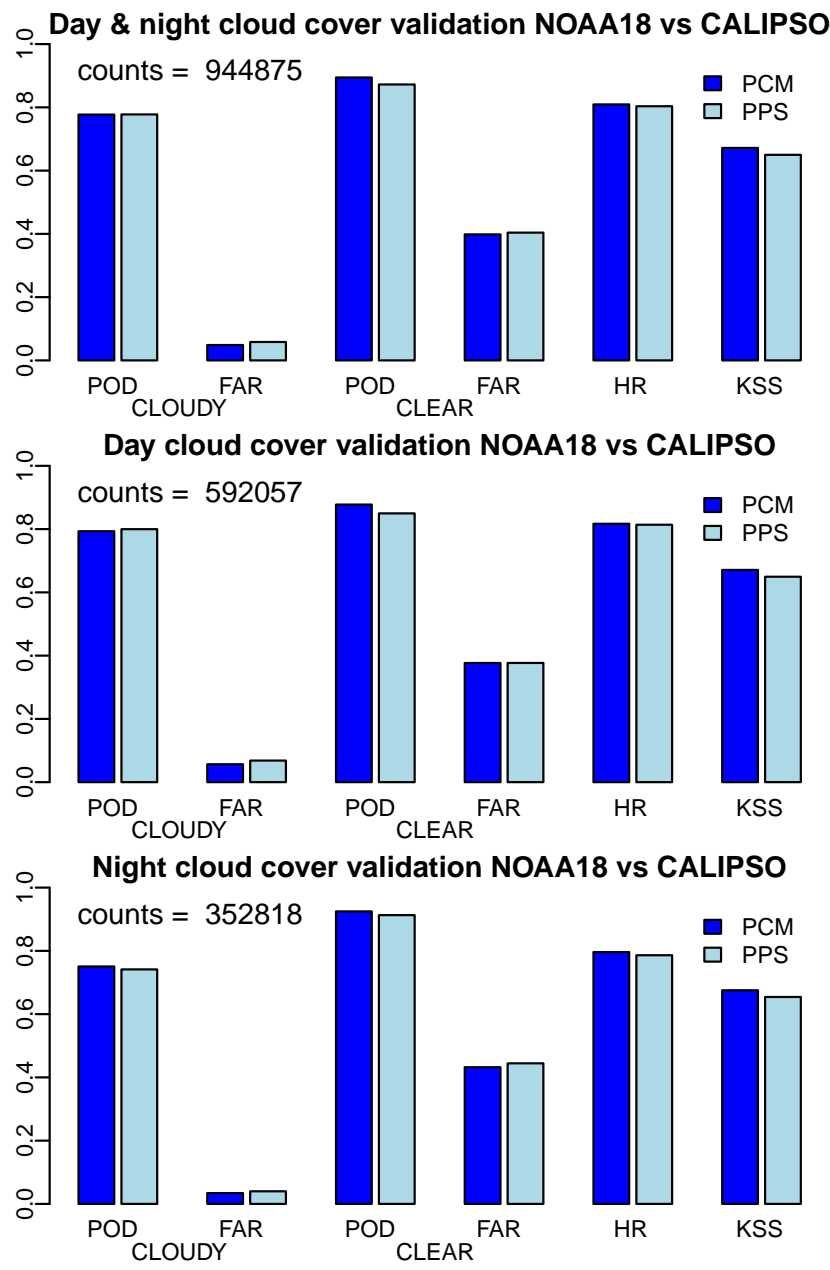

Fig. 18. Accuracy indicators of the PCM and PPS cloud detection skills derived from the comparison with CALIOP vertical feature mask. For details see Sect. 4.4.

The acquired results prove high cloud detection skills of the PCM method by reporting a good agreement with the reference data sets. On average PCM detects $4.4 \%$ less clouds than MOD/MYD35 products (Fig. 12a), while in the case of the PPS cloud mask the differences are even lower, from -0.5 to $0.9 \%$ (Fig. 6). Similar results were reported by Heidinger et al. (2002), who found that for the 3 selected satellite scenes MODIS cloud masks contained 1 to $3 \%$ more clouds than the CLAVR (Stowe et al., 1999) cloud mask derived from the LAC AVHRR data. These negative discrepancies decrease with the SZA for the MOD35 data (Fig. 12c), whereas the opposite trend is associated with the PCM-PPS differences which eventually become positive (Fig. 7a, e and i). This inconsistency might be partially related to the fact that MODIS cloud mask reports more clouds over snow than PCM (Fig. 12b), while PPS reports less (Fig. 8). Therefore, during the cold season associated with high SZA an absolute PCM-MOD35 difference increases. Regarding the sensor viewing geometry, the 
comparison against MODIS data is not conclusive as both instruments (AVHRR and MODIS) measure the same area under different angles. Therefore, such an analysis was performed only with the PPS algorithm, which relies on the same AVHRR input data as the PCM. The distribution of total cloud cover differences as a function of satellite zenith angle features a characteristic saw-shape pattern (Fig. 7b, f and $\mathrm{j}$ ), which originates from the division of angles' values into sectors. It can be presumed that without this partitioning the differences between PCM and PPS methods would rise continuously and the overall discrepancies between the algorithms would be higher. The relationship of the differences with the Sun-satellite relative azimuth angle does not reveal any significant pattern, and the noticeable variations might be attributed to the quantity of available observations (Fig. 7c, $\mathrm{g}$ and $\mathrm{k}$ ). However, the distribution of total cloud cover discrepancies related to the thermal contrast between SKT and the $10.8 \mu \mathrm{m}$ BT has a distinct drop of values within the 0 to $10 \mathrm{~K}$ range for both PPS and MODIS comparisons (Figs. 7b, $f, j$ and $12 d)$. This effect could be partially associated with the different approach towards local radiance variation analysis over water bodies, where PPS and MOD35 mark a pixel as cloudy whenever the spectral variance in its vicinity (e.g. $5 \times 5$ window) is sufficiently high (Di Vittorio and Emery, 2002). However, this implies that some of the clear-sky pixels around cloud edges with small thermal contrast are misclassified. In the PCM method the textural analysis is performed differently by means of the kernel convolution with a small window (Eq. 1), which assures that only pixels at cloud edges are detected. Therefore, the PPS and MOD35 products report more clouds than PCM over water bodies (Figs. 8 and 12b), which in turn modifies the overall results significantly, as it is the most extensive land cover class. This is also confirmed by the spatio-temporal analysis of the total cloud cover differences between the PCM and PPS methods (Fig. 11). For the latitude ranges of $40-50^{\circ}$ and $60-70^{\circ}$, where the water class is predominant over the study area, the PPS algorithm reports more clouds than PCM regardless of the time of the year. For other surfaces the total cloud cover difference remains negative in the case of the comparison with the MODIS data, whereas comparison with the PPS data, the PCM algorithm tends to report more clouds over the land surfaces excluding deserts. The same relationships could be observed on the annual total cloud cover composites for the NOAA18 satellite (Figs. 9 and 10). Moreover, the daytime PPS composite contains artefacts associated with areas characterised by the unreliably frequent cloud cover (up to $100 \%$ ) over Africa and Spain. They are related to land cover classes described by Anderson (1976) which do not resolve well the local spectral characteristics and lead to overestimation of the cloud amount. These areas are less apparent on the PCM total cloud cover composite because the PCM algorithm utilises more detailed land cover categorisation (Bartholomé and Belward, 2005). Furthermore, the PPS-based training data set was corrected by an analyst before being utilised during the PCM development phase. Another noticeable spatial difference is associated with mountains (e.g. Alps), where PCM reports more clouds than PPS especially during the nighttime. This is related to the fact that in the PPS method the SKT- $10.8 \mu \mathrm{m}$ thermal contrast test features very cloud conservative threshold ( $22 \mathrm{~K}$ at night) over the rough topography areas. This was introduced to account for the temperature variability induced by the comparison of $1 \mathrm{~km} \times 1 \mathrm{~km}$ AVHRR data with lowresolution SKT estimates (Dybbroe et al., 2005a). Nevertheless, due to this relaxed threshold the SKT-10.8 $\mu \mathrm{m}$ test often indicates clear-sky conditions, which misleads the PPS algorithm, and results in lower cloud coverage over the mountainous regions.

In order to evaluate the accuracy of the PCM and PPS algorithms, the total cloud cover observations provided by the SYNOP stations (Fig. 16a) were compared to the PCM and PPS cloud fractions derived over the $30 \mathrm{~km}$ buffer zone around each station. This analysis involved creation of the contingency table and computation of the classification quality indicators (Eqs. 5-11). They are the same for both methods or slightly better in the case of the PCM results (Fig. 17). The small improvement over the PPS algorithm was gained by the correction of the PPS/MOD10A1 training data set applied by the analyst. High probability of detection $(>0.8)$ and low false alarm rate $(<0.1)$ for the cloudy conditions remain at the same level for both algorithms regardless of the time of the day. Lower detection skills for the clear-sky cases can be attributed to the fact that an observer at a SYNOP station may report clouds which are further than the $30 \mathrm{~km}$ distance (for more comprehensive explanation see Karlsson, 1995). Nevertheless, the overall performance of both algorithms represented by the Kuipers skill score is high and the differences between the selected satellites are inconsiderably small. Furthermore, these results are consistent with the CALIOP/AVHRR validation (see Sect. 4.4) from the qualitative perspective; however their absolute values are higher. This is due to the fact that computation of fractional cloud cover within the $30 \mathrm{~km}$ buffer zone increases the probability of cloud detection. On the other hand, the CALIOP/AVHRR validation was performed on a single pixel basis, which yields more accurate results. The high sensitivity of a lidar system to detection of thin clouds which are not visible for the AVHRR sensor is well apparent as a relatively high FAR $(>0.4)$ for the clear conditions. This is also the reason for the higher cloud amount in the cloud mask derived from the MODIS instrument which has 1.38 and $8.55 \mu \mathrm{m}$ channels that improve the detection of mid-level and high clouds (Ackerman et al., 1998). The acquired classification quality criteria for the CALIOP/PPS cloud mask comparison remain in a good agreement (within the $10 \%$ range) with the study of Karlsson and Dybbroe (2010), who performed the same analysis over the Artic region.

Within the $30 \mathrm{~km}$ buffer zones around each station the mean PCM cloud probability was analysed as a function of the SYNOP cloud amount (Fig. 16b-d). The acquired 
distribution feature a reasonable spread of values from $\sim 12 \%$ for the clear-sky conditions to $\sim 90 \%$ for the fully overcast sky, regardless of the time of the day and the AVHRR channel configuration. The acquired probability distribution remains in a good consistency with the SYNOP validation results for the POLarization and Directionality of the Earth's Reflectances (POLDER) cloud mask (Bréon and Colzy, 1999). Although in that case the distribution expressed the percentage of cloudy pixels as a function of the SYNOP cloud amount, the PCM probability estimates feature almost a binary distribution (Fig. 15), which fits well to the binary output of the POLDER cloud mask. The same characteristics of the cloud probability distribution were reported in other studies (Gómez-Chova et al., 2007; Heidinger et al., 2012). Its specific "U shape", where most of the pixels are classified as confident clear $0 \%$ or cloudy $100 \%$, is related to the high spectral contrast of clouds on a satellite image as compared to other surfaces (see red dots in Fig. 2). Most of the classification probability estimates significantly different than 0 or $100 \%$ represent pixels with fractional cloud and/or snow coverage, ice clouds overlaying a cold surface, and thin cirrus clouds. These cases are far less frequent than fully overcast or clear-sky pixels, which results in highly polarised probability distribution (Fig. 15).

The snow component of the PCM output was validated against the MOD10A1 daily composite, which is one of the few existing snow cover data sets matching the spatial and temporal resolution of the AVHRR data. Although the high accuracy of this product was proven (Hall and Riggs, 2007), some misclassification especially between snow and clouds or overestimation of snow in forests may influence the quality indicators for the PCM method (Eqs. 5-11). Moreover, it has to be taken into account while interpreting the results that NOAA16 data were collected during the year 2011 while NOAA17 and NOAA18 data originate from the year 2008. Thus, different snow cover conditions between these periods influence the computed statistics. In this analysis only the cloud-free areas were considered; therefore some pixels at snow cover edges (Fig. 5), which are often misclassified as clouds (Hall and Riggs, 2007), were not taken into account. The best agreement between PCM and MOD10A1 total snow cover estimates is reported for the NOAA17 satellite which has the $1.6 \mu \mathrm{m}$ channel activated. For the platforms with the $3.7 \mu \mathrm{m}$ channel constantly operating the discrepancies are more significant. Although the POD of snow is considerably higher for the NOAA17 platform (Fig. 14), the FAR indicator is on the same level for the NOAA18 satellite. Therefore, it can be concluded that the PCM classification results for the NOAA17 and 18 satellites are valuable in terms of the snow composite derivation. Nevertheless, the accuracy of the PCM snow cover classification is strongly dependent on the illumination conditions. It was found that for the NOAA17 and 18 satellites the absolute PCM-MOD10A1 differences increase significantly above the Sun zenith angle of $70^{\circ}$ (Fig. 13a, e and i). A similar value was reported by Solberg et al. (2010).
This effect is not that apparent for the NOAA16 satellite possibly due to the different snow conditions in 2011 as compared to 2008.

The comparison and validation of the PCM results against space-borne and ground data proved the high cloud detection skills. As far as the snow classification is concerned the overall accuracy of the algorithm is reasonable, but special precaution has to be taken for data generated without the $1.6 \mu \mathrm{m}$ channel, especially for the high Sun zenith angle acquisitions. The lack of reference probability estimates for cloudy/clear-sky, cloudy/snow or snow/clear-sky conditions allows only the comparison with the SYNOP cloud amount, which confirms the expected value spread with low probabilities $(\sim 15 \%)$ for the clear-sky conditions and high ones ( $\sim 90 \%)$ for the fully overcast sky. Finally, the PCM cloud and snow classification was proven to be valid for a broad range of environmental conditions across Europe and northern parts of Africa.

\section{Conclusions}

This study presents a robust algorithm for cloud and snow detection on AVHRR imagery which provides complementary probability estimates. Its unique design is based on the concept of a multidimensional information space implemented as an array with precomputed probability estimates, where each dimension corresponds to different spectral, angular and ancillary data combination. In order to construct this array, continuous data such as spectral measurements have to be binned using a set of intervals which do not have to be equally distributed. Another novel feature of the PCM is employment of the ICS transformation, which utilises the PCA to combine reflectance at $0.6,0.8$, and $1.6 / 3.7 \mu \mathrm{m}$ with the thermal difference between $10.8 \mu \mathrm{m}$ channel and skin surface temperature provided by a climate model. This reduces dimensionality of the information space, enhances spectral contrast of thin/broken clouds, and improves separation between snow cover and ice clouds. The proposed methodology features a unique set of merits such as the following:

- Derivation of classification probability between clearsky/cloudy, clear-sky/snow and snow/cloudy conditions. This suppresses the usage of two different algorithms separately suited for cloud and snow detection. Moreover, such a triple-class probability gives more flexibility in terms of derivation of binary product, where different probability thresholds (more or less conservative) can be applied.

- Utilisation of all available spectral and ancillary information in a single step to retrieve probability estimates from a multidimensional LUT. Such an approach resolves the problem of interpretation of divergent test results occurring in the decision-tree methods. 
- Specification of multiple, irregularly distributed binning values, which resolves the problem of selection of a single threshold value which should feature the highest discrimination skills for instantaneous image acquisition conditions.

- Derivation of the algorithm's parametrisation based on a training data set composed of collocated satellite measurements and clear-sky/snow/cloud classification originating from another algorithm (PPS in this study), another sensor (MOD10A1 in this study) or ground observations (e.g. SYNOP).

- Simple algorithm design, which allows easy inclusion/exclusion of features by adding/removing dimensions in the LUT.

The PCM classification accuracy was assessed and validated on the basis of an extensive set of a reference data consisting of the PPS and MOD35 cloud masks, CALIOP vertical feature mask, SYNOP cloud observations, and MOD10A1 daily snow composite. The reported cloud detection skills were high $(\mathrm{POD}>0.8$ and $\mathrm{FAR}<0.1)$ and remained at the same level or even better than the reference PPS algorithm (due to initial correction of the training data set). Furthermore, some artefacts related to areas with implausibly high cloud cover present in the PPS data were almost not apparent in the PCM classification. The snow detection skills derived on a basis of comparison with MOD10A1 product are moderate $(0.42<\mathrm{POD}<0.62$ and $0.08<$ FAR $<0.17$ ), with significantly better results for the NOAA17 satellite with the $1.6 \mu \mathrm{m}$ channel activated and for low Sun zenith angle acquisitions. The probability estimates for cloudy conditions were found to be directly proportional to the SYNOP cloud amount and feature a reasonable spread (from $\sim 12 \%$ for clear-sky conditions to $\sim 90 \%$ for fully overcast sky). Nevertheless, to fully validate the PCM product the intercomparison with other probabilistic classification algorithms such as the one included in the PATMOS-x package, as well as the modified version of the PPS algorithm, will be performed. The source code of the PCM method implemented in R, together with the test cases and the LUTs are provided on the web page http://pcm.r-forge.r-project.org/.

Acknowledgements. The authors gratefully acknowledge the Science and Technology S $+\mathrm{T}$ division of armasuisse for financial support of the project; the Satellite Application Facility on Support to Nowcasting \& Very Short Range Forecasting (NWC SAF), Satellite Application Facility on Numerical Weather Prediction (NWP SAF), and Pascal Brunel (Météo-France) for providing the AVHRR processing software; European Centre for Medium-Range Weather Forecasts (ECMWF) for providing the NWP and SYNOP data; Joint Research Centre of the European Commission for providing land cover data; USGS for providing DEM data; and NASA for providing MODIS and CALIOP data.

Edited by: A. Macke

\section{References}

Ackerman, S., Strabala, K., Menzel, W., Frey, R., Moeller, C., and Gumley, L.: Discriminating clear sky from clouds with MODIS, J. Geophys. Res., 103, 32-141, 1998.

Allen, R., Durkee, P., and Wash, C.: Snow/Cloud Discrimination with Multispectral Satellite Measurements, J. Am. Meteorol., 29, 994-1004, doi:10.1175/15200450(1990)029<0994:SDWMSM>2.0.CO;2, 1990.

Anderson, J. R.: A land use and land cover classification system for use with remote sensor data, Vol. 964, United State Printing Office, Washington, 1976.

Arya, S., Mount, D., Netanyahu, N., Silverman, R., and Wu, A.: An optimal algorithm for approximate nearest neighbor searching fixed dimensions, J. ACM, 45, 891-923, 1998.

Bartholomé, E. and Belward, A.: GLC2000: a new approach to global land cover mapping from Earth observation data, Int. J. Remote Sens., 26, 1959-1977, 2005.

Bernstein, R.: Sea surface temperature estimation using the NOAA 6 satellite advanced very high resolution radiometer, J. Geophys. Res.-Oceans, 87, 9455-9465, 1982.

Bréon, F.-M. and Colzy, S.: Cloud detection from the spaceborne POLDER instrument and validation against surface synoptic observations, J. Appl. Meteorol., 38, 777-785, 1999.

Derrien, M. and Le Gléau, H.: MSG/SEVIRI cloud mask and type from SAFNWC, Int. J. Remote Sens., 26, 4707-4732, 2005.

Di Vittorio, A. V. and Emery, W. J.: An automated, dynamic threshold cloud-masking algorithm for daytime AVHRR images over land, IEEE T. Geosci. Remote, 40, 1682-1694, 2002.

Dybbroe, A., Karlsson, K., and Thoss, A.: NWCSAF AVHRR cloud detection and analysis using dynamic thresholds and radiative transfer modeling, Part I: Algorithm description, J. Appl. Meteorol., 44, 39-54, 2005a.

Dybbroe, A., Karlsson, K., and Thoss, A.: NWCSAF AVHRR cloud detection and analysis using dynamic thresholds and radiative transfer modeling, Part II: Tuning and validation, J. Appl. Meteorol., 44, 55-71, 2005 b.

Eyre, J., Brownscombe, J., and Allam, R.: Detection of fog at night using Advanced Very High Resolution Radiometer (AVHRR) imagery, Meteorol. Mag., 113, 266-271, 1984.

Fraser, R. and Kaufman, Y.: The relative importance of aerosol scattering and absorption in remote sensing, IEEE T. Geosci. Remote, 23, 625-633, 1985.

Gómez-Chova, L., Camps-Valls, G., Calpe-Maravilla, J., Guanter, L., and Moreno, J.: Cloud-screening algorithm for ENVISAT/MERIS multispectral images, IEEE T. Geosci. Remote, 45, 4105-4118, 2007.

Hall, D. and Riggs, G.: Accuracy assessment of the MODIS snow products, Hydrol. Process., 21, 1534-1547, 2007.

Hall, D., Riggs, G., Salomonson, V., DiGirolamo, N., and Bayr, K.: MODIS snow-cover products, Remote Sens. Environ., 83, 181194, 2002.

Heidinger, A. K., Anne, V. R., and Dean, C.: Using MODIS to estimate cloud contamination of the AVHRR data record, J. Atmos. Ocean. Tech., 19, 586-601, 2002.

Heidinger, A. K., Evan, A., Foster, M., and Walther, A.: A naive Bayesian cloud-detection scheme derived from CALIPSO and applied within PATMOS-x, J. Appl. Meteorol. Clim., 51, 11291144, 2012. 
Houghton, J., Meiro Filho, L., Callander, B., Harris, N., Kattenburg, A., and Maskell, K.: Climate change 1995: The science of climate change: contribution of working group I to the second assessment report of the Intergovernmental Panel on Climate Change, Vol. 19390, Cambridge University Press, 1996.

Hüsler, F., Fontana, F., Neuhaus, C., Riffler, M., Musial, J., and Wunderle, S.: AVHRR Archive and Processing Facility at the University of Bern: A comprehensive 1-km satellite data set for climate change studies, EARSeL eProceedings, 10, 83-101, 2011.

Hüsler, F., Jonas, T., Wunderle, S., and Albrecht, S.: Validation of a modified snow cover retrieval algorithm from historical 1-km AVHRR data over the European Alps, Remote Sens. Environ., 121, 497-515, 2012

Inoue, T.: On the temperature and effective emissivity determination of semi-transparent cirrus clouds by bi-spectral measurements in the $10 \mu \mathrm{m}$ window region, J. Meteorol. Soc. Jpn., 63, 88-99, 1985.

Jones, M., Saunders, M., and Guymer, T.: Reducing cloud contamination in ATSR averaged sea surface temperature data, J. Atmos. Ocean. Tech., 13, 492-506, 1996.

Karlsson, K.-G.: Estimation of cloudiness at high latitudes from multispectral satellite measurements, Ambio, 24, 33-40, 1995.

Karlsson, K.-G. and Dybbroe, A.: Evaluation of Arctic cloud products from the EUMETSAT Climate Monitoring Satellite Application Facility based on CALIPSO-CALIOP observations, Atmos. Chem. Phys., 10, 1789-1807, doi:10.5194/acp-10-17892010, 2010.

Kaufman, Y. J., Remer, L. A., Tanre, D., Li, R.-R., Kleidman, R., Mattoo, S., Levy, R. C., Eck, T. F., Holben, B. N., Ichoku, C., Martins, J. V., and Koren, I.: A critical examination of the residual cloud contamination and diurnal sampling effects on MODIS estimates of aerosol over ocean, IEEE T. Geosci. Remote, 43, 2886-2897, 2005.

Key, J. and Barry, R.: Cloud cover analysis with Arctic AVHRR data: 1. Cloud detection, J. Geophys. Res.-Atmos., 94, 18521$18535,1989$.

Khlopenkov, K. and Trishchenko, A.: SPARC: New cloud, snow, and cloud shadow detection scheme for historical 1-km AVHHR data over Canada, J. Atmos. Ocean. Tech., 24, 322-343, 2007.

Lyapustin, A., Wang, Y., and Frey, R.: An automatic cloud mask algorithm based on time series of MODIS measurements, J. Geophys. Res., 113, D16207, doi:10.1029/2007JD009641, 2008.

Mardia, K. V., Kent, J. T., and Bibby, J.: Multivariate AnalysisAcademic Press, New York, London, 1979.

Martins, J. V., Tanré, D., Remer, L., Kaufman, Y., Mattoo, S., and Levy, R.: MODIS cloud screening for remote sensing of aerosols over oceans using spatial variability, Geophys. Res. Lett., 29, 8009, doi:10.1029/2001GL013252, 2002.

Mayaux, P., Bartholomé, E., Fritz, S., and Belward, A.: A new landcover map of Africa for the year 2000, J. Biogeogr., 31, 861-877, 2004.

Merchant, C., Harris, A., Maturi, E., and MacCallum, S.: Probabilistic physically based cloud screening of satellite infrared imagery for operational sea surface temperature retrieval, Q. J. Roy. Meteorol. Soc., 131, 2735-2755, 2005.

Minnis, P., Trepte, Q. Z., Szedung, S. M., Chen, Y., Doelling, D. R., Young, D. F., Spangenberg, D. A., Miller, W. F., Wielicki, B. A., Brown, R. R., Gibson, S. C., and Geier, E. B.: Cloud detection in nonpolar regions for CERES using TRMM VIRS and Terra and Aqua MODIS data, IEEE. T. Geosci. Remote, 46, 3857-3884, 2008.

Nordhausen, K., Oja, H., and Tyler, D.: Tools for Exploring Multivariate Data: The Package ICS, J. Stat. Softw., 28, 1-31, 2008.

Pavolonis, M. J.: Advances in extracting cloud composition information from spaceborne infrared radiances-A robust alternative to brightness temperatures, Part I: Theory, J. Appl. Meteorol. Clim., 49, 1992-2012, 2010.

Pincus, R., Platnick, S., Ackerman, S., Hemler, R., and Patrick Hofmann, R.: Reconciling simulated and observed views of clouds: MODIS, ISCCP, and the limits of instrument simulators, J. Climate, 25, 4699-4720, 2012.

Plummer, S.: The GLOBCARBON Cloud Detection System for the Along-Track Scanning Radiometer (ATSR) Sensor Series, IEEE T. Geosci. Remote, 46, 1718-1727, 2008.

Rossow, W. and Garder, L.: Cloud detection using satellite measurements of infrared and visible radiances for ISCCP, J. Climate, 6 , 2341-2369, 1993.

R-project team: A Language and Environment for Statistical Computing, available at: http://www.R-project.org/ (last access: 1 January 2014), R Foundation for Statistical Computing, Vienna, Austria, 2012

Saunders, R. and Kriebel, K.: An improved method for detecting clear sky and cloudy radiances from AVHRR data, Int. J. Remote Sens. 9, 123-150, 1988.

Saunders, R., Matricardi, M., and Brunel, P.: A fast radiative transfer model for assimilation of satellite radiance observationsRTTOV-5, European Centre for Medium-Range Weather Forecasts, Reading, UK, 1999.

Schaaf, C. B., Gaoa, F., Strahlera, A. H., Luchtb, W., Lia, X., Tsanga, T., Strugnella, N. C., Zhanga, X., Jina, Y., Mullerc, J.-P., Lewisd, P., Barnsleye, M., Hobsone, P., Disneyd, M., Robertsd, G., Dunderdalec, M., Dollc, C., d'Entremontf, R. P., Hug, B., Liangh, S., Privettei, J. L., and Roy, D.: First operational BRDF, albedo nadir reflectance products from MODIS, Remote Sens. Environ., 83, 135-148, 2002.

Simpson, J. J. and Stitt, J. R.: A procedure for the detection and removal of cloud shadow from AVHRR data over land, IEEE Trans. Geosci. Remote Sens., 36, 880-897, 1998.

Simpson, J., McIntire, T., Stitt, J., and Hufford, G.: Improved cloud detection in AVHRR daytime and night-time scenes over the ocean, Int. J. Remote Sens., 22, 2585-2615, 2001.

Solberg, R., Wangensteen, B., Metsämäki, S., Nagler, T., Sandner, R., Rott, H., Wiesmann, A., Luojus, K., Kangwa, M., and Pulliainen, J.: GlobSnow Snow Extent Product Guide Product Version 1.0, European Space Angency, Finland, 2010.

Stowe, L., Davis, P., and McClain, E.: Scientific basis and initial evaluation of the CLAVR-1 global clear/cloud classification algorithm for the Advanced Very High Resolution Radiometer, J. Atmos. Ocean. Tech., 16, 656-681, 1999.

Tian, B., Azimi-Sadjadi, M., Vonder Haar, T., and Reinke, D.: Temporal updating scheme for probabilistic neural network with application to satellite cloud classification, IEEE T. Neural Netw., 11, 903-920, 2000

Tyler, D. E., Critchley, F., Dümbgen, L., and Oja, H.: Invariant coordinate selection, J. Roy. Stat. Soc. B, 71, 549-592, 2009. 
Uddstrom, M., Gray, W., Murphy, R., Oien, N., and Murray, T.: A Bayesian cloud mask for sea surface temperature retrieval, J. Atmos. Ocean. Tech., 16, 117-132, 1999.

Vemury, S., Stowe, L., and Anne, V.: AVHRR pixel level clear-sky classification using dynamic thresholds (CLAVR-3), J. Atmos. Ocean. Tech., 18, 169-186, 2001.

Vermote, E., Tanré, D., Deuze, J., Herman, M., and Morcette, J.: Second simulation of the satellite signal in the solar spectrum, 6S: An overview, IEEE T. Geosci. Remote, 35, 675-686, 1997.

Wang, M. and Shi, W.: Estimation of ocean contribution at the MODIS near-infrared wavelengths along the east coast of the US: Two case studies, Geophys. Res. Lett., 44, 3196-3205, doi:10.1109/TGRS.2006.876293, 2005.

Wang, M. and Shi, W.: Cloud masking for ocean color data processing in the coastal regions, IEEE T. Geosci. Remote, 44, 31963105, 2006.
Warren, D.: AVHRR channel-3 noise and methods for its removal, Int. J. Remote Sens., 10, 645-651, 1989.

Yang, Y., Di Girolamo, L., and Mazzoni, D.: Selection of the automated thresholding algorithm for the Multi-angle Imaging SpectroRadiometer Radiometric Camera-by-Camera Cloud Mask over land, Remote Sens. Environ., 107, 159-171, 2007.

Yhann, S. R. and Simpson, J. J.: Application of neural networks to AVHRR cloud segmentation, IEEE T. Geosci. Remote, 33, 590604, 1995.

Zhao, T., Guo, W., and Fu, C.: Calibrating and evaluating reanalysis surface temperature error by topographic correction, J. Climate, 21, 1440-1446, 2008. 